\title{
1 Diverse DNA modification in marine prokaryotic and viral communities
}

3 Satoshi Hiraoka $^{1 *}$, Tomomi Sumida ${ }^{1}$, Miho Hirai $^{2}$, Atsushi Toyoda ${ }^{3}$, Shinsuke Kawagucci ${ }^{2}$, Taichi Yokokawa ${ }^{2}$, 4 and Takuro Nunoura ${ }^{1}$

6 1. Research Center for Bioscience and Nanoscience (CeBN), Japan Agency for Marine-Earth Science and Technology (JAMSTEC), 2-15 Natsushima-cho, Yokosuka, Kanagawa 237-0061, Japan

8 2. Institute for Extra-cutting-edge Science and Technology Avant-garde Research (X-star), Japan Agency for Marine-Earth Science and Technology (JAMSTEC), 2-15 Natsushima-cho, Yokosuka, Kanagawa 2370061, Japan

11 3. Advanced Genomics Center, National Institute of Genetics, 1111 Yata, Mishima, Shizuoka 411-8540, Japan

13 Email: hiraokas@jamstec.go.jp 


\section{Abstract}

Chemical modifications of DNA, including methylation, play an important role in prokaryotes and

17 viruses. However, our knowledge of the modification systems in environmental microbial communities,

18 typically dominated by members not yet cultured, is limited. Here, we conducted 'metaepigenomic' analyses

19 by single-molecule real-time sequencing of marine microbial communities. In total, 233 and 163 metagenomic

20 assembly genomes (MAGs) were constructed from diverse prokaryotes and viruses, respectively, and 220

21 modified motifs and 276 DNA methyltransferases (MTases) were identified. Most of the MTases were not

22 associated with the defense mechanism. The MTase-motif correspondence found in the MAGs revealed 10

23 novel pairs, and experimentally confirmed the catalytic specificities of the MTases. We revealed novel

24 alternative motifs in the methylation system that are highly conserved in Alphaproteobacteria, illuminating the

25 co-evolutionary history of the methylation system and host genome. Our findings highlight diverse

26 unexplored DNA modifications that potentially affect the ecology and evolution of prokaryotes and viruses. 


\section{Introduction}

DNA chemical modifications are found in diverse prokaryotes and viruses as well as eukaryotes.

DNA methylation, a representative DNA modification, is catalyzed by DNA methyltransferase (MTase), whereas S-adenosylmethionine (SAM) provides a methyl group (1). In prokaryotes, three types of methylation (i.e., N6-methyladenine (m6A), 5-methylcytosine (m5C), and N4-methylcytosine (m4C)) have been investigated in detail (2). DNA methylation plays a role in the regulation of gene expression and mismatch DNA repair (3-5). Subsequently, the systems function on physiological machineries, including asymmetric cell division (6, 7), ultraviolet (UV) tolerance (8), motility (9), and virulence of pathogens (10-12). DNA methylation also facilitates cell protection from invasion of extracellular DNA, such as viruses and plasmids, known as restriction-modification (RM) systems (13). To overcome the defense system, some viruses possess MTases and modify their genomic DNA to escape the host RM system (2). Moreover, frequent gene duplication, loss, and horizontal gene transfer within and between domains have been revealed during the evolution of prokaryotes (14). In addition to methylation, other epigenetic modifications, such as phosphorothioate, have recently been shown to have a significant effect on cells, including maintenance of cellular redox homeostasis and epigenetic regulation (15). Research interests in various epigenomic systems in diverse prokaryotes and viruses are increasing because of their importance in microbial physiology, genetics, evolution, and disease pathogenicity (16-18). However, current knowledge of prokaryotic and viral epigenomics mostly relies on studies with culturable strains, although the majority of microbes have not yet been cultured.

The recent development of single-molecule real-time (SMRT) sequencing technology, one of the feasible methods to detect DNA modification, and its implementation in PacBio sequencing platforms, has revealed an array of DNA modifications of prokaryotic (19-24) and viral strains $(25,26)$. The capability of long reads with few context-specific biases (e.g., GC bias) (27) allows circler consensus sequencing (CCS) method to generate highly accurate high-fidelity (HiFi) reads through error collection with multiple 'subreads' sequences in every single read (28). Based on the innovative SMRT sequencing technique, we applied cultureindependent shotgun metagenomic and epigenomic analyses on freshwater microbial communities to reveal the vast DNA modification systems in nature, and established 'metaepigenomics' (29). Apart from PacBio, nanopore sequencing platforms produced by Oxford Nanopore Technologies (ONT) achieve longer reads that potentially improve metagenomic assembly with high diversity (30). Thus, a hybrid approach with $\mathrm{HiFi}$ and ONT reads is an ideal way to improve metaepigenomic analysis with accurate identification of modifications from organisms in highly diverse microbial communities. 
Here, we conducted a metaepigenomic analysis of pelagic microbial communities using SMRT sequencing technology to reveal the epigenomic characteristics of diverse marine prokaryotes and viruses whose epigenomic states have not been well described. The diverse DNA modifications were successfully characterized on numerous metagenomic assembled genomes (MAGs) from both prokaryotes and viruses obtained by a combination of PacBio Sequel, ONT GridION, and Illumina Miseq sequencing platforms. Our computational prediction and experimental assay confirmed several MTases responsible for the detected methylated motifs, including novel ones. In particular, a highly conserved methylation system with varied specificity was found in Alphaproteobacteria, suggesting co-evolution between the methylation systems and their host genomes.

Results and discussions

\section{Seawater sampling}

Four seawater samples were collected from epipelagic (5 and $90 \mathrm{mbsl})$ and mesopelagic (200 and 300 mbsl) layers from two closely located stations in the Pacific Ocean (referred to as CM1_5m, Ct9H_90m, CM1_200m, and Ct9H_300m) (Fig. S1a, Table S1). The hydrographic properties were distinct between the epipelagic and mesopelagic zones (31) (Fig. S1b). Subsurface chlorophyll $a$ maximum appeared at 60-100 mbsl during the samplings. The densities of prokaryotic cells and virus-like particles were highest in the uppermost layer and decreased with increasing water depth. The virus-to-prokaryote ratio increased with increasing water depth.

\section{Shotgun sequencing}

PacBio Sequel produced 16-21 million (75-104 Gb) subreads from each sample (Table S2). The CCS analysis produced 0.66-1.1 million HiFi reads with $>99 \%$ accuracy and average lengths ranging from 4311 to 4926 bp (Figs. S2a-d). The HiFi reads were estimated to cover 42-63\% of the community diversity in each sample (Fig. S3). In addition to the SMRT sequencing, we conducted shotgun sequencing of CM1_5m using GridION and obtained 25 million $(67 \mathrm{~Gb}$ ) ONT reads (Table S2). The average length of the ONT reads ( $2734 \pm 2013 \mathrm{bp}$ ) was comparatively shorter than the HiFi reads with high deviation, likely due to the methods used for DNA extraction based on bead-beating technique, although N50 reached $3.5 \mathrm{~kb}$ and the longest read 
Taxonomic assignment of the HiFi reads was performed using Kaiju (32) with the NCBI nr database (33) (Figs. S4a-c), and $83-89 \%$ and 3-12\% of the HiFi reads were assigned to Bacteria and Archaea, respectively. Only $2-8 \%$ and $0.4-3.7 \%$ of HiFi reads were assigned to Eukaryota and Viruses, respectively. Assignment ratios of prokaryotes were $85-91 \%, 31-74 \%$, and $21-53 \%$ at the phylum, order, and genus levels, respectively. Similar assignment was obtained by using the Global Ocean Reference Genomes Tropics (GORG-Tropics) database, which is composed of single-cell amplified genomes from pelagic surface layers (34) (Figs. S4d-f). Taxonomic compositions of full-length 16S rRNA gene sequences in the HiFi reads using BLASTN (35) against the SILVA database (36) were also consistent with the protein-based assignments using nr or GORG-Tropics (Figs. S4g-i). These similarities verify that the profile estimated using Kaiju with nr (Figs. S4a-c) is appropriate for further analysis.

Within the prokaryotes, Proteobacteria predominated in all samples at the phylum level (36-52\%). Cyanobacteria was the next most abundant phylum in the epipelagic waters, CM1_5m (19\%) and Ct9H_90m (27\%), where photosynthesis is active. In the mesopelagic CM1_200m and Ct9H_300m communities, Chloroflexi (8.0 and 8.6\%, respectively) and Thaumarchaeota (6.1 and 7.9\%) were the dominant phyla. Actinobacteria (4.4-5.0\%), Bacteroidetes (2.3-5.4\%), and Euryarchaeota (3.1-5.3\%) were abundant in all samples. The ratio of Archaea was relatively low in the epipelagic samples (3.3 and 5.1\%) while high in the mesopelagic samples (11.8 and 12.2\%). Within eukaryotes, the dominant phylum was Chlorophyta (0.2$3.2 \%)$, followed by Haptista $(0.2-1.2 \%)$ and Ascomycota (0.5-0.7\%). The highest abundance of eukaryotes was observed in Ct9H_90m from the chlorophyll $a$ maximum zone. Viral reads were abundant in the epipelagic samples (4.0-4.7\%) and scarce in the mesopelagic samples (0.66-0.67\%). Among the viruses,

110 Myoviridae was the most abundant family, followed by Siphoviridae, Phycodnaviridae, and Podoviridae.

111 Myoviridae, Siphoviridae, and Podoviridae belong to the order Caudovirales, known as prokaryotic viruses

112 (37). In contrast, Phycodnaviridae, a member of giant viruses with large capsids, primarily infects eukaryotic 113 algae, including members of Chlorophyta (38). Because major floating viruses passed through the used 0.22$114 \mu \mathrm{m}$ membrane filters, most of the viral reads were expected to be derived from lytic viruses replicating in host 115 cells, lysogens within host genomes, or extracellular giant viruses. Viruses without double-stranded DNA (i.e., 116 single-stranded DNA and RNA viruses) were not observed due to the experimental method employed.

117 Overall, the taxonomic compositions were consistent with those of previous studies (39-42). 
REBASE Gold Standard database (43). Generally, genes assigned to MTase (M), REase (R), and protein fused with the MTase and REase domains (RM) showed similar compositions among the microbial communities, and their relative abundances decreased slightly with increasing water depth (Fig. 1a). Within the MTase proteins (i.e., M and RM), Type II predominated, accounting for 76.5-78.6\% in each sample (Fig. 1b). The relative abundances of Type I (11.2-12.1\%) and III (3.4-5.9\%) were approximately 2-3 times lower than those identified in the genomes of prokaryotic isolates, reported as $27 \%$ and $8 \%$, respectively (20). Among the detected MTases, the most abundant modification type was m6A (56.9-62.9\%), followed by m4C (15.6-19.6\%) and m5C (14.6-19.6\%) (Fig. 1c).

\section{Metagenomic assembly and genome binning}

The HiFi and ONT reads were assembled into 7829-29391 contigs (Table S3, Fig. S5). The total length of the assembled contigs from the HiFi reads (Ct9H_90m, CM1_200m, and Ct9H_300m) ranged from $83-123 \mathrm{Mb}$, the $\mathrm{N} 50$ values were $11-13 \mathrm{~kb}$, and the length of the longest contigs was $308-546 \mathrm{~kb}$. In contrast, the contigs of CM1_5m assembled from ONT reads after polishing showed outperformed statistics that reached a total length of $524 \mathrm{Mb}$ with a $29 \mathrm{~kb}$ N50 value, likely due to the number of ONT reads longer than the HiFi reads.

The contigs were binned into a total of 233 prokaryotic MAGs (P-MAGs) (Table S3, Data S1). Among the contigs of CM1_5m, 1165 (3.96\%) were assigned to 130 P-MAGs. From Ct9H_90m, CM1_200, and Ct9H_300m, 1009 (12.9\%), 3366 (31.1\%), and 2761 (33.9\%) contigs assembled from the HiFi reads were binned into 30, 41, and 32 P-MAGs, respectively. In total, 9.4-31.5\% of the HiFi reads were re-mapped to the P-MAGs for each sample. The completeness of the P-MAGs was $28.6 \%$ on average, and the highest value was $98.8 \%$. The estimated contamination levels were low (1.2\%, on average). N50 ranged from $6.4 \mathrm{~kb}$ to 1.42 Mb. Subread coverages ranged from 31.4-3305.7× per P-MAGs, and 218 P-MAGs (94\%) showed >50×, which is sufficient to detect $\mathrm{m} 6 \mathrm{~A}$ and $\mathrm{m} 4 \mathrm{C}$ modifications using SMRT sequencing according to the manufacture's instruction.

A total of 233 P-MAGs spanned 14 phyla (Data S1). Among the P-MAGs, we predicted full-length 16S rRNA gene sequences from 64 P-MAGs. No assignment was obtained for 10 (4.3\%) and 56 (24\%) PMAGs at the phylum and class levels, respectively, likely owing to the existence of vast phylogenetically undescribed lineages. A total of 110 (47\%) P-MAGs belonged to the phylum Proteobacteria, including 41 and 52 P-MAGs assigned to the class Alphaproteobacteria and Gammaproteobacteria, respectively. Most of the 
150 Alphaproteobacteria P-MAGs were assigned to the order Candidatus ( $\mathrm{Ca}$.) Pelagibacterales, known as

151 SAR11, which is one of the most abundant groups in pelagic ecosystems (44). P-MAGs affiliated to other 152 dominant phyla such as Chloroflexi (18 P-MAGs); Ca. Marinimicrobia (18 P-MAGs), Acidobacteria (16 P153 MAGs), Bacteroidetes (15 P-MAGs), Verrucomicrobia (5 P-MAGs), and Cyanobacteria (5 P-MAGs) were 154 also identified. Among the Chloroflexi P-MAGs, six 16S rRNA gene sequences were identified, all of which were assigned to SAR202 clade. P-MAGs assigned to Archaea were composed of two phyla: Euryarchaeota (27 P-MAGs) and Thaumarchaeota (3 P-MAGs).

In addition to the prokaryotic MAGs, a total of 163 viral MAGs (V-MAGs) were retrieved from the contigs (Data S1). Most (98\%) of the V-MAGs were retrieved from CM1_5m, while three, one, and zero VMAGs were from Ct9H_90m, CM1_200m, and Ct9H_300m, respectively. This distinct difference between CM1_5m and the others likely resulted from the variance of virus abundance and the outstanding efficiencies of metagenomic assembly using ONT reads only available from CM1_5m (Figs. S4a, S5, and Table S3). The lengths of the V-MAGs ranged from 8-248 $\mathrm{kb}$ and $64 \mathrm{~kb}$ on average. Subread coverages ranged from 1.5$894.8 \times$ and 103 P-MAGs (63\%) were $>50 \times$. In total, $4.72 \%$ of the HiFi reads were re-mapped to the V-MAGs in CM1_5m. Nine V-MAGs were identified as proviruses. The most abundant order was Siphoviridae (26 VMAGs), followed by Myoviridae (14 V-MAGs), Phycodnaviridae (2 V-MAGs), and Podoviridae (2 VMAGs), while most of the 119 (73\%) V-MAGs were not given order-level taxonomy because of the limited genomic data of environmental viruses in the reference database.

\section{Metaepigenomic analysis}

A total of 178 and 42 candidate modified motifs were detected in 108 (46\%) P-MAGs and 15 (9\%) V-MAGs, respectively (Data S2). Mapped subread coverages of the modified motifs were compatible with PMAGs and V-MAGs that ranged from 30.6 to $508.9 \times$ and 88.3 to $568.8 \times$, respectively. The detected motifs were composed of 59 unique motifs, including 32 motifs with palindromic sequences that allow double-strand modification. Among the unique motifs, 27 and 23 were classified as m6A and m4C methylation types, respectively. Although the current SMRT sequencing technology does not support the detection of the m5C motif, we found four candidate m5C motifs with high subread mean coverages ( $259 \times$ on average). Among the methylated motifs from P-MAGs, 57 (35\%) showed $<50 \%$ modification ratios on the genome, possibly because of the weak detection power of modification from subreads or the existence of strain-level epigenomic heterogeneity in the microbial communities. The modification types of the other 5 motifs were unclassified and possibly represented chemical modifications out of the above three methylations, such as 
181

phosphorothioation (24). The unclassified motifs showed low modification ratios (ranging from 14-45\% with $30 \%$ on average), similar to previous observations of phosphorothioated motifs in Escherichia coli (12\%) and Thaumarchaeota (20\%) strains $(22,23)$.

Among the P-MAGs with methylated motifs, GATC was detected most frequently (41 P-MAGs),

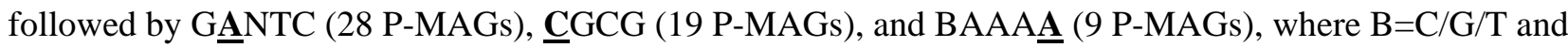
$\mathrm{N}=\mathrm{A} / \mathrm{C} / \mathrm{G} / \mathrm{T}$, and the underlined boldface indicates methylation sites. Among the V-MAGs, RG$\underline{\mathbf{C}}$ ( $(9 \mathrm{~V}-$ MAGs) was the most abundant motif, followed by $\underline{\mathbf{C}} \mathrm{CNGG}$ (4 V-MAGs), GGW $\underline{\mathbf{C}}$ (3 V-MAGs), and GGH드 (3 V-MAGs), where $\mathrm{R}=\mathrm{A} / \mathrm{G}, \mathrm{Y}=\mathrm{C} / \mathrm{T}, \mathrm{W}=\mathrm{A} / \mathrm{T}$, and $\mathrm{H}=\mathrm{A} / \mathrm{C} / \mathrm{T}$. It is worth noting that, even considering some vague motifs, at least 15 motifs (i.e., BAAA므, ACAA $\underline{\mathbf{A}}, \mathrm{CAA} \underline{\mathbf{A}}$ T, CT$\underline{\mathbf{A}} \mathrm{G}, \mathrm{G} \underline{\mathbf{A}} \mathrm{TGG}$,

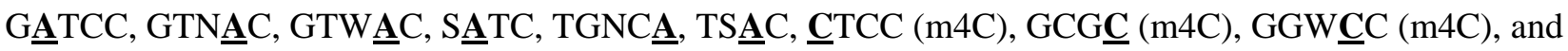
TGGCCA (m5C), where $\mathrm{S}=\mathrm{C} / \mathrm{G}$ ) did not match the known recognition sequences of MTases in REBASE repository. In addition, motifs likely catalyzed by Type I MTases, which are generally characterized as bipartite sequences with a gap of unspecified nucleotides (e.g., ATGNNNNNTAC), were undetected in all the P-MAGs and V-MAGs. This result indicates that Type I RM systems were scarce in the epipelagic and mesopelagic prokaryotes and viruses. Regarding vertical distribution of the modified motifs along with the water column, no clear relationship between the frequency of the motif and their habitats was observed; 0.65 , $1.2,0.76$, and 0.84 motifs were detected on average in CM1_5m, Ct9H_90m, CM1.5m_200m, and Ct9H_300m P-MAGs, respectively.

\section{Prediction of MTases and corresponding methylated motifs}

To identify MTases that catalyze methylation of the detected motifs, systematic annotation of MTase genes was performed. Sequence similarity searches against known genes stored in REBASE (43) identified 171, 43, and 7 of M, R, and RM genes, respectively, from 112 (48\%) P-MAGs (sequence identities ranged 20-92\%) (Data S3). Among the M and RM genes from P-MAGs, m6A (64\%) was the most abundant MTase type, followed by $\mathrm{m} 4 \mathrm{C}(14 \%)$ and $\mathrm{m} 5 \mathrm{C} \mathrm{(10 \% ),} \mathrm{as} \mathrm{found} \mathrm{in} \mathrm{the} \mathrm{HiFi} \mathrm{read} \mathrm{analysis} \mathrm{(Fig.} \mathrm{1c).} \mathrm{Among} \mathrm{the}$ MTase types, Type II MTases were the most abundant (82\%), while 9\% and 6\% genes showed the highest sequence similarity to Type I and III MTases, respectively. This trend is also consistent with the HiFi reads analysis that Type I and III MTases were scarcely detected in the communities (Fig. 1b). Only three genes encoded DNA sequence-recognition proteins, known as the S subunit in the Type I RM system. Most of the MTases were orphan and only four pairs of Type II MTase and REase gene were predicted to possess the 
211 same motif sequence specificity and were adjusted on the genome, which may constitute intact Type II RM

212 systems. Other known antiviral defense systems associated with DNA modification—BREX (45) and

213 DISARM (46) were surveyed; however, no MTase genes likely associated with these systems were found

214 through the P-MAGs. Overall, our analyses highlighted the previously unknown diverse MTases in epipelagic

215 and mesopelagic prokaryotic communities and suggest that the methylation systems play unexplored roles

216 apart from the known defense mechanisms of exogenous DNA.

A total of 58 (20\%) MTase genes from P-MAGs showed the best sequence similarity to MTases, whose specificity was exactly matched to the motif identified in our metaepigenomic analysis (Data S2, S3).

219 For example, CM1_200m.P15 contained one MTase that showed the best sequence similarities to those that 220 recognized C․ㅗGG, which was perfectly congruent with the motif detected from P-MAG. For

221 CM1_200m.P39, two MTases similar to those that recognize either TTA $\underline{\mathbf{A}}$ or $\underline{\mathbf{C} G C G}$ were identified, and 222 these motifs were congruently detected in the genome. In Ct9H_300m.P17, five MTases were predicted, two

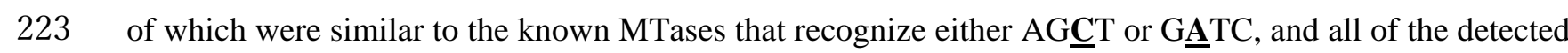
224 methylated motifs in the genome were completely matched, suggesting that the two MTases were active while 225 the other three were inactive.

In contrast, at least one methylated motif was detected in 40 (17\%) P-MAGs, while no MTase gene was found. We assumed that the corresponding MTase genes were missed because of insufficient genome completeness or that these MTase genes diverged considerably from known MTase genes. At least one MTase gene was found in 44 (19\%) P-MAGs, but no methylated motifs were detected. We anticipate that the MTase genes were inactive, or the corresponding methylated motif was undetected due to the low sensitivity of SMRT sequencing, especially in m5C modification $(19,20)$. (30\%) V-MAGs (sequence identities ranged 23-73\%) (Data S3). Similar to the case of P-MAGs, Type II

234 MTases were the most abundant (79\%), followed by Type I (7\%), and no Type III MTase was detected 235 through the V-MAGs. In contrast to P-MAGs, m4C (62\%) was the most abundant modification type in V236 MAGs, followed by m6A (30\%) and m5C (1\%). All the MTases and methylated motifs were unmatched in V-

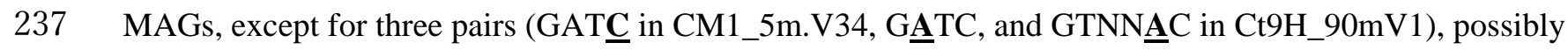
238 because of the very few viral MTases stored in REBASE Gold Standard database, where currently 16 viral 239 MTase genes were found in a total of 1938 MTase genes. 


\section{Exploration and experimental verification of MTases with new methylated motifs}

Among the detected MTase genes, 132 (74\%) and 94 (96\%) MTases from P-MAGs and V-MAGs, respectively, showed inconsistency between the recognition motifs of their closest relatives and the methylated motifs identified in our metaepigenomic analysis (Data S2, S3). The result suggested that the homology-based estimation of MTase specificity was not sufficient, as in our previous metaepigenomic study of the freshwater microbiome (29). To reveal the catalytic specificity of these MTases, we investigated potential pairs of MTase and methylated motif as follows: 1) MTase and methylated motifs were present in the same genome and novel correspondence was estimated; 2) modification types (i.e., m4C, m5C, and m6A) of MTase and methylated motifs were concordant, and 3) the complete sequence of the MTase gene was retrieved. Subsequently, methylation specificities of the selected MTases were experimentally verified with heterologous expression in E. coli (Data S4). Briefly, plasmids with one artificially synthesized MTase gene were constructed and transformed into E. coli cells, and the methylation status of the isolated plasmid DNA was subsequently observed by REase digestion after heterologous expression. Viral MTases were not selected for the experiment to improve the efficiency of heterologous expression.

In Actinobacteria, Ct9H_300m.P26, one m6A MTase gene, and two m6A and m4C motifs were detected, but none of the MTase and motif matched each other. Thus, we predicted that Ct9H300mP26_1870, whose closest homolog encoded an MTase that exhibits CTCGAGG methylation activity, would encode an MTase that recognizes BAAA므, whereas the motif sequence was not registered in REBASE and no MTase was currently reported to recognize the motif. The REase digestion assay was consistent with the hypothesis that ScaI (AGTACT specificity) did not cleave the BAAA $\underline{A} G T A C T$ sequence, which overlapped with BAAAA and AGTACT sequences, on the plasmids only when MTase was expressed in the cells (Fig. 2a). We named this protein M.AspCt9H300mP26I, as a novel MTase that possesses BAAA $\underline{\mathbf{A}}$ specificity.

In Actinobacteria, Ct9H_90m.P5, two MTase genes, and three methylated motifs were detected, and a pair of MTase and motif was concordantly matched, whereas the other MTases did not match any motifs. The latter MTase gene Ct9H90mP5_10800 showed moderate sequence similarity (32\%) with a low E-value (1e-70) to M.AspCt9H300mP26I using BLASTP search, and either of the remaining motifs was m6A and m4C. Thus, we predicted that Ct9H90mP5_10800 MTase, whose closest homolog is an m6A MTase that exhibits ATTA프 methylation, would have BAAA모 specificity. As expected, the REase digestion assay showed that ScaI did not cleave the BAAA expressed (Fig. 2b). Thus, we named this protein M.AspCt9H90mP5I, as a novel MTase that possesses BAAA $\underline{\mathbf{A}}$ specificity. We should note that another candidate MTase gene, Ct9H90mP30_5500, detected in 
272 Actinobacteria Ct9H_90m.P30 was estimated to possess the same BAAA프 specificity and showed moderate

273 (33\%) and high (87\%) sequence similarities to M.AspCt9H300mP26I and M.AspCt9H90mP5I, respectively, 274 although the protein was insolubilized in E. coli, resulting in no clear cleavage inhibition in our experiment.

A Planctomycetes CM1_200m.P2 had three MTase genes and two methylated motifs. One of the MTases showed the highest sequence similarity to those recognizing TTA프 with high similarity (64\%). The other CM1200mP2_32760 and CM1200mP2_5150 MTases showed the highest sequence similarity to those catalyzing m6A modification and recognizing GTTA $\underline{\mathbf{A} C}$ and ATTA스, respectively, with low similarity (37\% and 25\%, respectively). The two detected motifs were GCG $\underline{\mathbf{C}}(\mathrm{m} 4 \mathrm{C})$ and CAA $\underline{\mathbf{A} T}$ (m6A), the latter of which was not found in REBASE. Thus, we expected that either or both MTases would recognize and methylate the novel CAAㅁT motif. The construct CM1200mP2_32760 was not successfully prepared in our experiment, likely because the protein was toxic to E. coli. In contrast, CM1200mP2_5150 MTase showed that MluCI (AATT specificity) did not cleave all sequence sites CAAㅁTT on the plasmids only when MTase was expressed, clearly indicating that MTase recognizes CAA $\underline{\mathbf{A} T}$ (Fig. 2c). Accordingly, we named the protein M.PspCM1200mP2I as a novel MTase that possesses a previously unknown CAA_AT specificity.

Chloroflexi CM1_5m.P129 had one MTase gene, which showed the highest sequence similarity to those recognizing TCTAGA (whose modification type and position were not reported). However, the only methylated motif detected in the genome was ACAA $\underline{\mathbf{A}}$, which no MTase was currently reported to recognize. Thus, we hypothesized that CM15mP129_7780 MTase should recognize and modify this novel motif. The REase digestion assay was consistent with the hypothesis that BceAI (ACGGC specificity) did not cleave the sequence site ACAA므CGCG only when MTase was expressed (Fig. 2d). Accordingly, we named this protein M.CspCM15mP129I, as a novel MTase that possesses previously unreported ACAA $\underline{\mathbf{A}}$ specificity.

In Ca. Marinimicrobia CM1_200m.P10, one MTase gene, and one methylated motif were detected. The reported recognition motif of the closest MTase is GAAGA (the modified base is the second position of

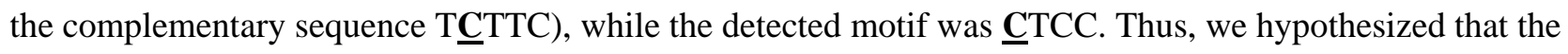

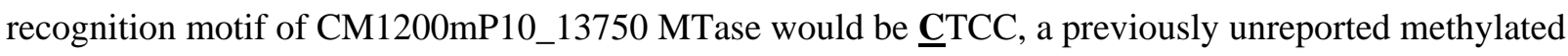
motif. The REase digestion assay showed that ScaI was inhibited to cleave the GGAGTACTCC sequence site,

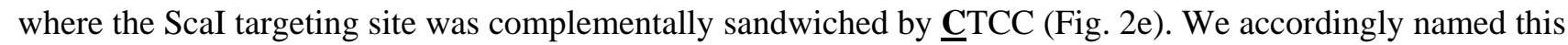
protein M.MspCM1200mP10I. 
Furthermore, we conducted a re-sequencing analysis to examine the methylation status of the chromosomal DNA of $E$. coli that each novel MTase gene was transformed and expressed. As a result, two

BAAA $\underline{\mathbf{A}}, \mathrm{CAA} \underline{\mathbf{A} T}$, ACAA $\underline{\mathbf{A}}$, and $\underline{\mathbf{C} T C C}$ were successfully recalled in the genomes of E. coli (Table S4).

\section{Phylogenetic distribution of modified motifs}

To investigate the phylogenetic distribution of the DNA modification system in the MAGs, we used 117 P-MAGs (>20\% completeness) and all 163 V-MAGs for robust phylogenetic tree reconstruction, and visualized the modification ratios of the detected motifs in each genome (Fig. 3). Within the P-MAGs, modified motifs were sporadically distributed across the phyla, whereas some showed great concordance with the phylogenetic clades. For example, within the phylum Actinobacteria, $\underline{\mathbf{C} G C G}$ and BAAA$\underline{\mathbf{A}}$ were spread in all genomes of the class Acidimicrobiia but were not detected in the class Actinobacteria. In contrast, A $\underline{\mathbf{A} T T}$ was found in three P-MAGs belonging to a subclade in Acidimicrobiia. TTA $\underline{\mathbf{A}}$ was found in four P-MAGs in

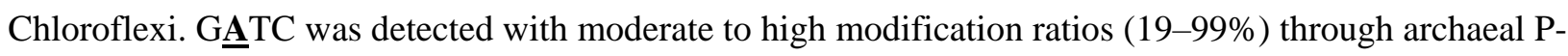
MAGs with two exceptions; no significant GㅅTC signature was detected in Euryarchaeota Ct9H_90m.P24 (7\%) and CM1_5m.P82 (0.4\%) possibly due to the weak or absent methylation activity in the host organisms. AG $\underline{\mathbf{C} T}$ was observed in all two Thaumarchaeota P-MAGs with high modification ratios (82-91\%). $\underline{\mathbf{C} G C G}$ was found in members from three phyla across the domain: Actinobacteria, Chloroflexi, and Euryarchaeota.

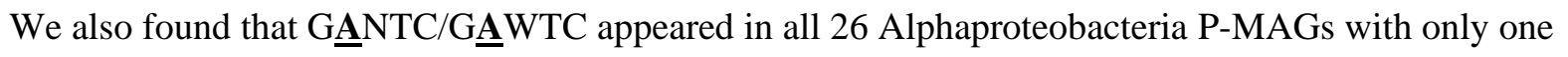
exception, indicating great conservation of the methylation systems in the group as described below. Other than the methylation, AGCT modified motif showed weak modification ratios (2-19\%) through the class $\mathrm{Ca}$. Poseidoniia P-MAGs, although the motif was detected only in Ct9H_300m.P10 in motif prediction analysis.

This result emphasizes that the phylogeny-based modification ratio analysis is efficient for analyzing infrequently modified motifs.

In sharp contrast, many motifs showed no clear associations with the phylogenetic topology. For example, GWGC appeared solitary with high modification ratio in Chloroflexi CM1_200m.P6. Similarly, 
330 insufficient coverage depth for modification detection. The 125 P-MAGs spread in diverse phyla, such as

331 Proteobacteria, Bacteroidetes, $\mathrm{Ca}$. Marinimicrobia, Chroloflexi, Gemmatimonadetes, Cyanobacteria, and

332 Verrucomicrobia. Interestingly, neither methylated motifs nor MTase genes were detected in P-MAGs

333 belonging to several lineages: all two members of Gemmatimonadetes, all two of Nitrospinae, and all five of

334 Verrucomicrobia (Data S1). Methylated motifs were also absent from all three Deltaproteobacteria P-MAGs,

335 although two of them possessed the MTase gene. Within the Gammaproteobacteria P-MAGs, 31 of 52

336 genomes lacked both methylated motifs and MTase genes. Taken together, these facts suggest the absence of

337 a DNA methylation system in several clades, which is in contrast to a previous study reporting pervasiveness

338 of DNA methylation among bacteria and archaea (20). Although further study is required, these observations

339 imply the unexplored benefits of the absence of DNA methylation.

Methylated motifs were occasionally detected with low modification ratios in most V-MAGs,

341 except for Phycodnaviridae and Myoviridae (Fig. 4). Among the Phycodnaviridae V-MAGs, Ct9H_90m.V1

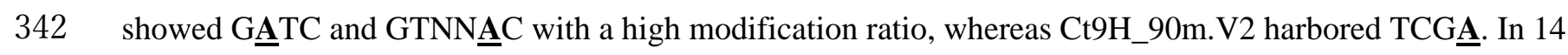

343 Myoviridae V-MAGs, 0-5 methylated motifs were detected. However, the proteomic tree showed numbers of

344 V-MAGs that were not assigned but closely related to the Myoviridae family (referred to as 'Myoviridae-

345 like'). The Myoviridae-like V-MAGs appeared to frequently share several motifs (e.g., RG두, $\underline{\mathbf{C}} \mathrm{CWGG}$,

346 GGW $\underline{\mathbf{C}}$ ) with different combinations among them and sometimes harbor additional motifs, while a few

347 numbers of modified motifs were detected in the motif prediction analysis ( 0.95 motifs per genome on

348 average). This indicates that the taxonomic assignment of the viral genome was frequently missed due to the

349 lack of viral genomes in reference database and the severe underestimation of modified motifs in V-MAGs,

350 likely due to their small genome size (see Materials and Methods). Note that the methylated motifs detected in

351 the V-MAGs were scarcely shared with those in the P-MAGs. No modified motifs other than methylation was

352 found in the V-MAGs. Five Myoviridae-like V-MAGs were predicted to be proviruses, although no clear

353 difference was observed in the modification ratio from the other non-provirus V-MAGs. In 39 Myoviridae-

354 like V-MAGs, several MTases were encoded in their genomes (ranging from 0-8 and 2.4 MTase genes per

355 genome on average), while scarcely detected in the other V-MAGs (ranging from 0-3 and 0.1 on average)

356 (Data S3). 


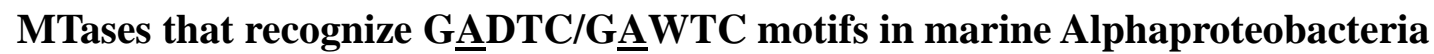

GANTC methylation is considered to be well conserved in Alphaproteobacteria and is assumed to play an important role in cell cycle regulation via gene regulation (47). Indeed, G $\underline{A} N T C$ was previously identified in diverse lineages of Alphaproteobacteria isolates $(20,48,49)$ and one MAG (29) using the modern SMRT sequencing technique, and no alternative motifs have been reported. In our metaepigenomic analysis, GㅁNTC was concordantly detected in 26 of 40 Alphaproteobacteria P-MAGs (Data S3). In addition, we

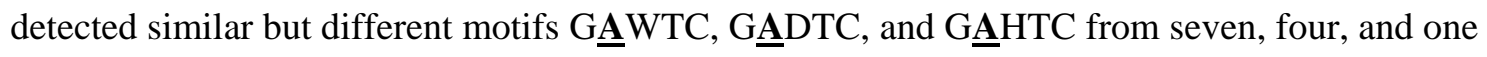
Alphaproteobacteria P-MAGs, respectively (where D=A/G/T) (Fig. S6). This result strongly suggests the presence of unknown variations in the methylation system in the lineage. From the Alphaproteobacteria PMAGs, we predicted 13 complete gene sequences of MTase that were assumed to recognize either of the motifs. However, all of them showed the highest sequence similarity to those known to recognize G with high sequence similarity (47\%-80\%) (Data S3).

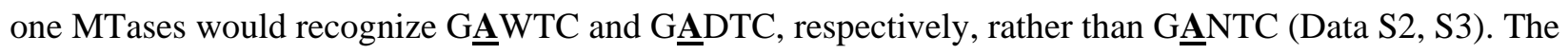
REase digestion assay of the former four MTases (CM15mP30_3110, CM15mP57_4380, CM15mP70_4410, and CM15mP111_3240) showed that TfiI (GAWTC specificity) cleavage was completely blocked only when MTase was expressed in the cells, whereas HinfI (GANTC specificity) partly cleaved the plasmids (Figs. S7ad). Despite exhibiting off-target effects under high concentrations of the enzyme, known as 'star activity' in REases $(50,51)$, assays of purified CM15mP111_3240 MTase protein suggested that its canonical specificity was G్WTC (see Note S1, Figs. S8a-c). The digestion pattern in the assay of CM15mP20_30 was also congruent with the hypothesized G_ADTC methylation, and re-sequencing analysis successfully recalls the methylated motif (Note S2, Fig. S7e, Table S4). In contrast, as expected, robust GㅅNTC specificity was confirmed in the assay of CM15mP16_9820, which completely inhibited both TfiI and HinfI cleavage (Fig. S7f). Accordingly, we named the four (M.PspCM15mP30I, M.AspCM15mP57I, M.PspCM15mP70I, and M.RspCM15mP111I) and one (M.AspCM15mP20I) proteins as novel MTases that preferentially recognize

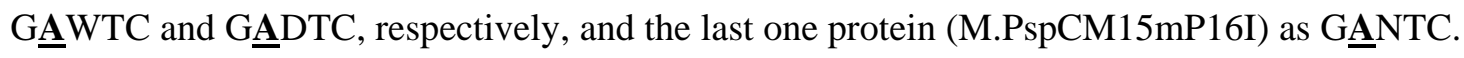
G ANTC specificity, is one of the model proteins of prokaryotic MTase $(20,47,52)$. Based on the sequence alignment of the 13 MTases with M.CcrMI and its homologs, a glycine residue (corresponding to Gly40 in M.CcrMI) was roughly conserved in all MTases with G $\underline{A}$ TC specificity, while it was replaced with lysine or aspartic acid in all with G $\underline{\mathbf{A} W T C}$ (Fig. S9). It has been reported that the M. CcrMI protein contains a 
substructure that forms a pocket to accommodate the third position of the recognized motif (i.e., nucleotide ' $\mathrm{N}$ ' in GANTC); two hydrophobic residues, Leu38-Leu42 stacks, and flexible Gly39 and Gly40 allow the acceptance of variable nucleotides in the position (53). This led to the hypothesis that a replacement of lysine/aspartic acid with glycine at the bottom of the fitting pocket would trigger physical interference in the

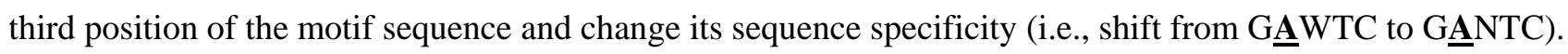
To test this hypothesis, we constructed a substitution mutant D49G of CM15mP111_3240 (the position corresponding to M.CcrMI Gly40) and conducted the REase digestion assay. However, the mutant showed partial inhibition of HinfI cleavage as compatible with the original MTase, suggesting that another factor rather than merely Gly40 residue defined the third position of the motif as 'W' (Fig. S8d).

\section{Evolutionary history of methylation systems in Alphaproteobacteria}

G $\underline{A}$ TC/G $\underline{A} D T C / G \underline{A} W T C$ motifs in Alphaproteobacteria, we analyzed the phylogenetic diversity of the methylated motifs and the frequencies of the motif sequences on each Alphaproteobacteria P-MAGs (Figs. 5a and c). In Rhodospirillales, SAR116, and Rhodobacteraceae P-MAGs, all four subsets of G $\underline{A}$ NC (i.e.,

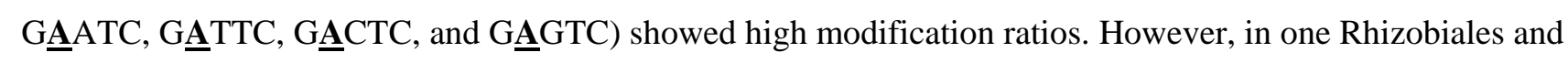

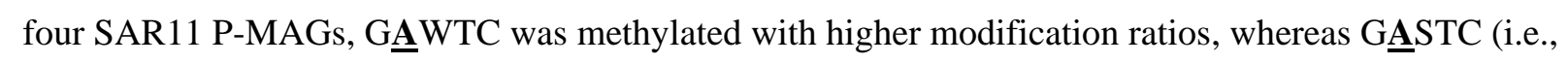

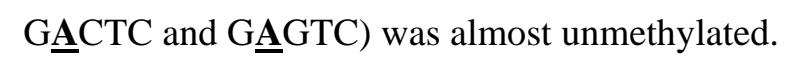

The phylogenetic topologies of P-MAGs and MTase were matched in Rhodospirillales, SAR116, Rhodobacteraceae, and Rhizobiales, suggesting good conservation of the MTases in these clades (Figs. 5a and d). In contrast, those in SAR11 showed incongruence with them, possibly because of the weak robustness of the phylogenetic inference of the MTases supported by low bootstrap values. Neither

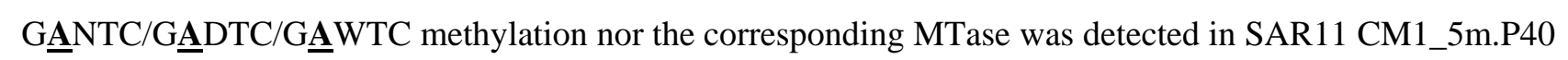
(Data S2 and S3), indicating that the organism lacked the methylation system. Regardless of the inconsistent topologies between organisms and proteins, MTases likely comprise a monophyletic group. Thus, it is suggested that the methylation systems have been maintained in Alphaproteobacteria and the MTases with

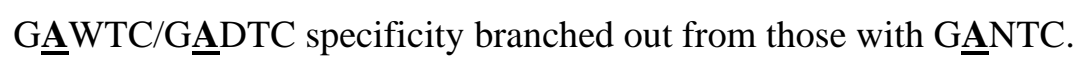
sequences were methylated (Figs. 5b and c). In Rhodospirillales, SAR116, and Rhodobacteraceae P-MAGs, in which G $\underline{A}$ NC was highly methylated, $\log _{2}$ Observed/Expected ratio (O/E ratio) of all subset of GANTC sequence was $-1.14 \pm 0.53$ (s.d.) on average. This means that GANTC sequences present with $>2$-fold lower frequency than expected from random distribution on their genomes, suggesting the existence of negative 
pressure against GANTC sequences. In contrast, in Rhizobiales and SAR11 P-MAGs, except for

CM1_5m.P40, the GAWTC O/E ratio was significantly lower than that of GASTC $(-1.73 \pm 0.59$ and $0.43 \pm$ and concordantly the $\mathrm{O} / \mathrm{E}$ ratios were approximately zero $(-0.09 \pm 0.08)$, suggesting a weak or no selective pressure under GANTC sequence on the genome. Alphaproteobacteria class, we calculated the O/E ratios using 112 and 195 accessible genomes that covered diverse Alphaproteobacteria (54) and all major subclades (I-V) of SAR11 (44, 55), respectively (Data S5). All constituent sequences of GANTC generally showed negative O/E ratios in Rhodospirillales, Sphingomonadales, Rhizobiales, Caulobacterales, and Rhodobacterales (-1.78 \pm 0.59$)$ (Figs. S10a and b). In contrast, those of Rickettsiales and Holosporales, including numbers of endosymbiotic members, were temperate $(-0.39 \pm 0.20)$. Only in SAR11, O/E ratios of GAWTC were significantly lower than those of GASTC $(-1.30 \pm 0.57$ and $-0.14 \pm 0.37$, respectively) ( $p<0.05$, U-test, Bonferroni correction), and this was concordant with the P-MAG analysis (Fig. 5b). These results indicated that all constituent sequences of GANTC were under negative pressure in Alphaproteobacteria, except Rickettsiales and Holosporales with weak pressure, and SAR11 with selectively attenuated pressure in GASTC constituents. Thus, the O/E ratio profile implied that the GANTC methylation system was not strictly conserved in all Alphaproteobacteria, rather than the GEWTC methylation system maintained in the exceptional group.

The estimated phylogenetic tree of SAR11 showed that one and seven P-MAGs belonged to subclades IV and V, respectively (Fig. S10c). The O/E ratios of GAWTC were significantly negative (-1.30 \pm $0.45)$ in contrast to those of GASTC $(0.09 \pm 0.30)$ ( $p<0.05$, U-test), but not evenly distributed throughout the SAR11 subclades (Fig. S10d). For example, the GAWTC O/E ratios were higher in subclade Ic (-0.58 \pm 0.10$)$, whereas those of GASTC were comparatively lower in subclade Ia.1 (-0.51 \pm 0.05$)$. Notably, the GAWTC $\mathrm{O} / \mathrm{E}$ ratios varied in subclade $\mathrm{V}$ (ranging from -2.7 to -0.1 ). Within subclade $\mathrm{V}$, five minor subclades were identified based on the phylogenetic topology associated with the $\mathrm{O} / \mathrm{E}$ ratio. One minor subclade, here we referred to Va, showed the lowest GAWTC O/E ratio (-1.83 \pm 0.59$)$ through the SAR11 subclades. In contrast, subclades $\mathrm{Vb}, \mathrm{Vc}$, and $\mathrm{Vd}$ showed comparatively higher GAWTC O/E ratios $(-0.58 \pm 0.28,-0.47 \pm 0.06$, and $0.62 \pm 0.04$, respectively). The other subclade, Ve, showed comparatively moderate $\mathrm{O} / \mathrm{E}$ ratios $(-1.00 \pm 0.42)$, which is compatible with other major subclades. Despite such variations, overall, the O/E ratio profile suggested that the negative selective pressure in the GAWTC sequence is highly conserved but not or weak in the GASTC sequence through the SAR11 subclades. This may be driven by DNA methylation caused by MTases with GAWTC specificity, and the fluctuating pressure among the subclades may be associated with ecological and evolutionary niches, although further investigation is required. 


\section{Metaepigenomics for exploring prokaryotic and viral DNA modification systems in the marine} environment.

Prokaryotic and viral DNA modification systems should play significant biological functions and

have been highlighted; however, little is known about their diversity, ecological role, and evolutionary history, especially in the environmental community. Several studies conducted bisulfite sequencing to investigate prokaryotic $\mathrm{m} 5 \mathrm{C}$ modifications using environmental samples $(56,57)$, but other $\mathrm{m} 6 \mathrm{~A}$ and $\mathrm{m} 4 \mathrm{C}$ modifications, which are more popular in prokaryotes, were not investigated. Community-level prokaryotic methylomes have been reported recently $(29,58,59)$, but the community-level viral methylome has not yet been reported.

The present study has conducted a first metaepigenomic analysis of pelagic microbial communities that dominated members not yet cultured with high complexity and successfully acquired unprecedented DNA modifications. From the HiFi read analysis, which was free from any biases induced in PCR or metagenomic assembly processes, MTase genes were distributed with a similar compositions of modification and MTase types through water depth (5-300 mbsl) (Fig. 1). The reconstructed P-MAGs and V-MAGs possessed a number of modified motifs, including novel ones (Data S2). Subsequent REase assay experiments identified 11 MTases responsible for these reactions, including five with novel specificity (Fig. 2, Fig. S7, and Data S3). The complex evolutionary history of the prokaryotic DNA methylation system is previously reported and considered to have resulted from repeated gene loss, duplication, and horizontal gene transfer, even across phylum or domain levels (14) as well as changes in MTases in sequence specificity (60). This possibility is supported by the solely presented motifs as well as linage-associated motif conservation from the marine prokaryotic and viral communities in this study (Figs. 3 and 4). Moreover, our analysis provided strong evidence that, in marine Alphaproteobacteria, the methylation systems changed their sequence specificity and affected genomic content through evolutionary history (Fig. 5). Consequently, these results demonstrated that metaepigenomics is effective for comparative methylation analysis within and between microbial populations, as well as modification analysis other than the popular methylations.

Meanwhile, the variance of DNA modifications among lineages has already been applied in bioinformatic applications. For example, an approach of metagenomic binning based on the methylation patterns of assembled contigs has been proposed (61-63). However, our results indicate that sets of methylated motifs are frequently shared within phylogenetically close lineages at even higher taxonomic levels such as phylum or order (Fig. 3), and thus could be worthless for distinguishing contigs into individual genome bins. 
From the other perspective, careful attention should be paid to the fact that metaepigenomic analysis is based on assembled 'consensus' genomes that may overlook epigenomic heterogeneity at lower taxonomic levels such as strain and species. Recent studies have reported possible variations in sets of methylated motifs and MTase genes at the genus to strain levels in wide prokaryotic lineages $(21,64-66)$. Resolving the strain-level diversity of DNA modifications in complex metagenomic samples remains a challenge.

\section{A possible function of DNA methylation in marine prokaryotic and viral communities}

The major detected MTase genes in P-MAGs were orphan and lacked a cognate REase gene, implying that most MTases in pelagic prokaryotes are inactive for protection against extracellular DNA and viral invasion via known physiological systems such as RM (67), BREX (45), and DISARM (46). The similar relative abundance of the MTase genes and their composition in the microbial communities (Fig. 1) suggest that the effects of environmental factors changing with water depth are limited. The possible role of the methylation systems is a factor involved in gene regulation. In addition, because solar UV radiation at the sea surface damages prokaryotic DNA $(68,69)$, some DNA methylations may function for DNA mismatch repair to exhibit photo stress tolerance. In E. coli, DNA methylation functions as a marker of the original (parental) DNA strand and facilitates mismatch repair on newly synthesized (daughter) unmethylated strands that frequently occur during cell division under high UV radiation $(4,5)$. It is anticipated that DNA methylation may play a key role in adaptation to the vast marine epipelagic and mesopelagic layers in prokaryotes, although further experimental and proteomic analyses (e.g., transcriptome and metatranscriptome) are required to confirm the epigenetic regulation of the genes involved.

Viruses, the most abundant biological entities in oceanic environments, play diverse roles in marine ecosystems (70). Among the V-MAGs, family level variance was found in the methylomes: in Myoviridae VMAGs, m4C motifs were frequently detected with several m5C and m6A motifs that encode numbers of MTase genes in their genomes; in Phycodnaviridae V-MAGs, several m6A motifs were detected as reported previously (71); in the other V-MAGs, including Siphoviridae and Podoviridae, methylation was scarcely detected. These results suggest the existence of strong selective pressure to maintain the methylation system in marine Myoviridae (and possibly Phycodnaviridae), but not in Siphoviridae and Podoviridae. Hence, DNA methylation in Myoviridae (and possibly Phycodnaviridae) may be associated with the genetic roles and ecological strategies of these groups, although the details remain uncertain. 
It has been discussed that the main advantageous function of viral MTases is a self-defense weapon against host-encoded defense systems $(25,72,73)$. However, this hypothesis was not concordant with our results that limited numbers of MTases constituted the known defense systems through the P-MAGs, as discussed above. Thus, the self-defense weapon may play a minor role in DNA methylation in marine viruses. One of the known roles of viral MTase is the initiation of DNA packaging during the late stages of viral infection, found in bacteriophage P1 (74). In this system, m6A modification labels ends of the concatemeric viral DNA molecules produced by rolling-circle replication, and the end points, where seven methylated motif sites are clustered in bacteriophage P1, are subsequently cut by an enzyme for DNA packaging into capsids. However, the Myoviridae V-MAGs possessed a variety of m4C motifs with different combinations in their genome (Fig. 4), and the features are likely inefficient to use methylation as a delimiter of concatenated viral DNA replicons. Another possible role of viral methylation is to increase the stability of DNA for dense packing within a viral capsid, as well as alpha-putrescinylthymine modification in bacteriophage $\varphi \mathrm{W}-14$ (75). In addition, several viral genes are known to be transcriptionally controlled by a self-encoded MTase originally found in bacteriophage P1 (76). The possibility that viral MTase regulates host gene expression to facilitate viral genome replication cannot be ruled out. It would be interesting to explore the role of DNA modification in the viral life cycle.

\section{Evolutionary history of methylation systems in Alphaproteobacteria} such as cell cycle master regulation, and its homologs are highly conserved in Alphaproteobacteria $(47,48)$. All of the known homologs in Alphaproteobacteria are believed to recognize G $\underline{A} N T C$, and the methylation system was acquired early in the evolution of Alphaproteobacteria (77). However, we found unprecedented

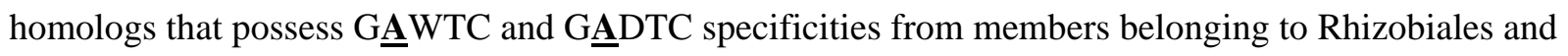
SAR11, and the specificities of these representatives were experimentally demonstrated (Notes S1 and S2, Figs. S6 and S7). Although the phylogenetic topology of the MTases does not match that of the SAR11

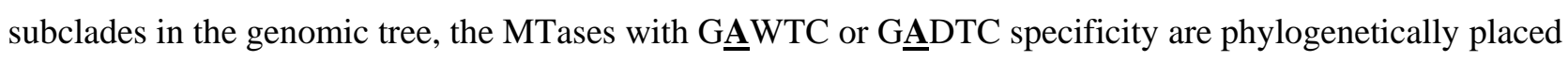
within those of GNAC with high sequence similarity. Thus, all MTases likely comprise a monophyletic group and share a common ancestor rather than acquired from distant lineages by horizontal gene transfer. In addition, it is assumed that the methylation systems have significant importance in the cell cycle process as M.CcrMI, and thus have been under strong selective pressure for maintenance in the orders. The MTase with

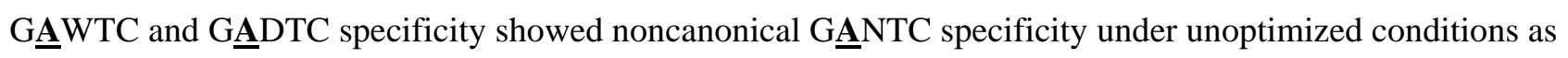
star activity (Notes S1 and S2, Fig. S7). This enzymatic feature resulted in a scenario in which these protein 
548 groups evolved from the ancestral MTase with GNANC specificity by depressing the affinity with GASTC or

549 GACTC sequences. The assay of a mutant protein in which we changed one residue at the bottom of the

550 pocket, which likely accommodated the third position of the motif and distinguished GANTC and GAWTC

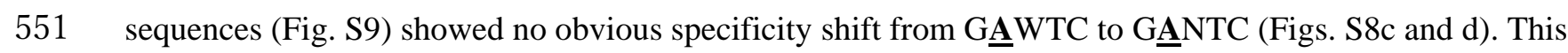

552 result suggests that the GASTC affinity is limited by other or additional residues, or the MTase with GAWTC

553 specificity forms a structure distant from M.CcrMI (53); thus, further consideration is required.

The O/E ratio analysis showed an oppressive profile of GAWTC sequence present on the genome compared with GASTC in the Rhizobiales and SAR11 P-MAGs. In contrast, GANTC were generally oppressive in the other Alphaproteobacteria P-MAGs, and these were concordant with the detected methylated motifs and specificity of the MTases (Fig. 5). This selection pressure suggests a significant (and may be harmful) effect of methylation on biological processes such as gene expression (i.e., a critical regulatory change driven by methylation at the internal gene coding sequence and/or promoter region). The low frequency of the GANTC sequence in Alphaproteobacteria genomes was previously reported; however, SAR11 (formerly classified in Rickettsiales) was not recognized as an individual group and the frequency of the GAWTC sequence was not evaluated (48). Here, our analysis drastically expands the knowledge about methylation in the class that at least a part of SAR11 members possesses the Ge्ATC methylation system. Furthermore, the O/E ratio analysis showed strong and specific negative pressure on the GAWTC sequence in their genomes, in contrast to the other Alphaproteobacteria orders (Fig. S10). Variance in GAWTC O/E ratios among SAR11 subclades showed that different methylation states were associated with the evolution of each subclade. Consequently, our findings provide novel insights into prokaryotic epigenomics that DNA methylation plays a greater role in host evolution than previously recognized. Despite the many challenges of culturing in marine prokaryotes, further investigations are required to evaluate the relationships among molecular function, ecological benefit, and evolution of M.CcrMI homologs in Alphaproteobacteria.

\section{Effects and challenges of metaepigenomics on environmental microbiology} prokaryotic and viral DNA modifications in diverse and complex pelagic microbial ecosystems. It is envisioned that metaepigenomics of prokaryotes and viruses under different ecological niches (e.g., sea area and water depth) and subjects (e.g., soil, gut, and symbionts) will significantly deepen our understanding of the effects of DNA modification and lead to various applications. 
HiFi reads covered only half of the microbial communities in this study (Fig. S3), and further sequencing efforts are required for higher-resolution analysis to reveal a whole picture of epigenome in environmental microbial communities. Despite the advantage of the hybrid approach of SMRT and ONT sequencing, each of them requires more than $10 \mu \mathrm{g}$ of DNA as initial input for library preparation, which allows us to conduct ONT sequencing only for a sample from the surface layer (CM1_5m). In addition, even using current SMRT sequencing technology, only a limited number of DNA modification types can be detected and classified with sufficient reliability (i.e., m4C and m6A), although a number of modifications occur in nature (2). Further development of sequencing technology, accurate assembly tools, and reliable modification detection methods will be required for deeper evaluation of prokaryotic and viral DNA methylation in environments.

\section{Methods}

\section{Seawater sampling}

Seawater samples were collected at two close pelagic stations of Japan Agency for Marine-Earth Science and Technology (JAMSTEC) in the northwest Pacific Ocean during JAMSTEC KM19-07 cruises of the Research Vessel (R/V) Kaimei in September 2019 (Fig. S1, Table S1). The sampling stations were approximately 180 and $140 \mathrm{~km}$ offshore from the main island of Japan, and $60 \mathrm{~km}$ from each other. Each 90$300 \mathrm{~L}$ of seawater was collected from 5 and $200 \mathrm{mbsl}$ at station CM1 (34.2607 N 142.0203 E) and 90 and 300 mbsl at station Ct9H (34.3317 N 141.4143 E) (referred to as CM1_5m, CM1_200m, Ct9H_90m, and Ct9H_300m, respectively). Sampling permit for expeditions in Japan's exclusive economic zone was not required as in domestic areas and did not involve endangered or protected species. Seawater from 5 mbsl was directly sampled using a built-in pumping system from the bottom of the ship via approximately $5 \mathrm{~m}$ of intake pipe that was designed for continuous monitoring of sea surface hydrography. The valve of the pumping system was opened at least $30 \mathrm{~min}$ before starting the sampling to entirely flush the internal water and rinse the pipe. Seawaters from 90, 200, and 300 mbsl were sampled using 12-L Niskin-X bottles (General Oceanic, Miami, Florida, USA) in a CTD rosette system. The vertical profiles of temperature, salinity, and pressure data were obtained using the SBE9plus CTD system (Sea-Bird Scientific, Bellevue, Washington, USA). The vertical profiles of dissolved oxygen (DO) concentrations were obtained using an in situ DO sensor RINKOIII (JFE Advantech, Hyogo, Japan) connected to the CTD. The vertical profiles of chlorophyll $a$ concentrations were obtained using an in situ Fluorometer RINKO profiler (JFE Advantech). The seawater samples in the Niskin-X bottles were transferred to sterilized $20 \mathrm{~L}$ plastic bags and immediately stored at $4{ }^{\circ} \mathrm{C}$ until further filtration. Filtration was performed with $0.22-\mu \mathrm{m}$ Durapore membrane filters (Merck KGaA, 
611 Darmstadt, Germany) after prefiltration with $5 \mu \mathrm{m}$ Durapore membrane filters (Merck KGaA) onboard. The

612 filters were then immediately stored at temperatures lower than $-30{ }^{\circ} \mathrm{C}$.

\section{Flow cytometric assessments of prokaryotic cell and viral-like particle abundances}

Seawater samples for flow cytometric assessments of prokaryotic cell and viral-like particle (VLP) abundances were obtained every $10-50 \mathrm{~m}$ at station $\mathrm{CM} 1$ and $10-100 \mathrm{~m}$ at station $\mathrm{Ct} 9 \mathrm{H}$, fixed with $0.5 \%$ (w/v) glutaraldehyde (final concentration) in $2 \mathrm{~mL}$ cryo-vials on board and stored at $-80{ }^{\circ} \mathrm{C}$ until further analysis. For assessment of prokaryotic cell abundance, $200 \mu \mathrm{L}$ of each sample was stained with SYBR Green I Nucleic Acid Gel Stain (Thermo Fisher Scientific, Waltham, Massachusetts, USA) ( $\times 5$ of manufacturer's stock, final concentration) at room temperature for $>10 \mathrm{~min}$. For assessment of VLP abundance, $20 \mu \mathrm{L}$ of each fixed sample was diluted 10 times with TE buffer and stained with SYBR Green I $(\times 0.5$ of manufacturer's stock, final concentration) for $10 \mathrm{~min}$ at $80{ }^{\circ} \mathrm{C}$. Total prokaryotic cells and VLP abundance in $100 \mu \mathrm{L}$ samples were determined using an Attune NxT Acoustic Focusing Flow Cytometer (ThermoFisher Scientific) by their signature in a plot of green fluorescence versus side scatter $(78,79)$.

\section{DNA extraction and shotgun sequencing} according to the supplier's protocol. The filters were cut into 3-mm fragments and directly suspended in the extraction solution from the kit for cell lysis. SMRT sequencing was conducted using a PacBio Sequel system (Pacific Biosciences of California, Menlo Park, California, USA) at the National Institute of Genetics (NIG), Japan. SMRT libraries for HiFi read via CCS mode were prepared with a 5-kb insertion length. Briefly, 4-6 kb DNA fragments from each genomic DNA sample were extracted using the BluePippin DNA size selection system (Sage Science, Beverly, Massachusetts, USA). The SMRT sequencing library of CM1_5m and the other three samples were prepared using the SMRTbell Template Prep Kit 1.0-SPv3 and SMRTbell Express Template Prep Kit 2.0, respectively, according to the manufacturer's protocol (Pacific Biosciences of California). The final SMRT libraries were sequenced using four, three, three, and three Sequel SMRT Cell 1M v3 for CM1_5m, CM1_200m, Ct9H_90m, and Ct9H_300m, respectively. Nanopore sequencing of CM1_5m was conducted using a GridION Mk1 platform with five flow cells according to the manufacturer's standard protocols at NIG. ONT libraries were prepared and purified simultaneously by filtering out a small number of fragments using AMPure XP beads (Agencourt BioSciences, Beverly, Massachusetts, USA). 
641 Illumina sequencing ( $2 \times 300$ bp paired-end reads) was conducted using an Illumina MiSeq platform (Illumina, 642 San Diego, California, USA) at JAMSTEC. Illumina libraries were prepared using the KAPA Hyper Prep Kit 643 (Roche, Basel, Switzerland) and mixed with Illumina PhiX control libraries, as described previously (80).

\section{Bioinformatic analysis of sequencing reads and assembled genomes}

CCS reads that contained at least five full-pass subreads on each polymerase read and with $>99 \%$ average base-call accuracy were retained as HiFi reads using the standard PacBio SMRT software package with the default settings. Metagenomic coverage of HiFi reads was estimated using Nonpareil3 with default settings (81). For taxonomic assignment of HiFi reads, Kaiju (32) in Greedy-5 mode ('-a greedy -e 5' setting) with NCBI nr (33) and GORG-Tropics databases (34) were used. HiFi reads that potentially encoded $16 \mathrm{~S}$ ribosomal RNA (rRNA) genes were extracted using SortMeRNA (82) with default settings, and full-length 16S rRNA gene sequences were predicted using RNAmmer (83) with default settings. The 16S rRNA gene sequences were taxonomically assigned using BLASTN (35) against the SILVA database release 128 (84), where the top-hit sequences with e-values $\leq 1 \mathrm{E}-15$ were retrieved. Coding sequences (CDSs) with $>33$ aa length in HiFi reads were predicted using Prodigal (85) in anonymous mode ('-p meta' setting). For Illumina read data, both ends of reads that contained low-quality bases (Phred quality score $<20$ ) and adapter sequences were trimmed using TrimGalore (https://github.com/FelixKrueger/TrimGalore) with default settings. The remaining paired-end reads were merged with at least 10 bp overlap using FLASH (86) with default settings.

HiFi and ONT reads were de novo assembled using wtdbg2 (Redbean) with the settings for PacBio CCS and ONT reads, respectively, according to the provided instructions (87). Assembled contigs from ONT reads were polished using both $\mathrm{HiFi}$ and Illumina short reads and HyPo (88). For the polishing, $\mathrm{HiFi}$ and Illumina reads were mapped on the pre-polished contigs using pbmm2, an official wrapper software for minimap2 (89) with CCS reads settings, and Bowtie2 (90) with '-N 1' setting, respectively.

The assembled contigs were binned using MetaBAT (91) based on genome coverage and tetranucleotide frequencies as genomic signatures, where the genome coverage was calculated with Illumina reads using Bowtie2 with '-N 1' setting. The quality of bins was assessed using CheckM (92), which estimates completeness and contamination based on taxonomic collocation of prokaryotic marker genes with default settings. Bins with $<10 \%$ contamination were retrieved according to the metagenome-assembled genome (MIMAG) standards (93) and defined as prokaryotic metagenome assembled genomes (P-MAGs). We note 
671 that the partial genome would be sufficient for detecting DNA modifications and modified motifs;

672 completeness was not considered for P-MAG definition. Sequences of 16S rRNA genes in each P-MAG were 673 retrieved using RNAmmer (83) with default settings. The taxonomy of the P-MAGs was estimated based on 674 16S rRNA gene sequences, CAT (94), and Kaiju (32). P-MAGs that were not assigned to prokaryotes or assigned but with low reliance ( $<0.6$ supported score) using CAT were excluded from further analysis. CDSs with >33 aa length in each P-MAG were predicted using Prodigal (85) with default settings. Functional annotations were achieved through HMMER (95) searches against the Pfam database (96), with a cutoff evalue of $\leq 1 \mathrm{E}-5$.

For viral sequence collection, the assembled contigs were screened using VirSorter2 (97) with default settings. Quality assessment of the retrieved contigs and removal of flanking host regions from integrated proviruses was performed using CheckV (98). Contigs assigned to either 'Complete' or 'Highquality' or 'Medium-quality' were defined as viral metagenome assembled genomes (V-MAGs) and used for further analysis. Taxonomy levels lower than kingdom were estimated using CAT (94). CDSs were predicted using Prodigal (85) in an anonymous mode ('-p meta' setting). Functional annotations were achieved in the same way as for P-MAGs.

\section{Bioinformatic analysis of modification systems} according to the officially provided tool SMRT Link v8.0. Briefly, subreads were mapped to the assembled contigs using pbmm2, and the interpulse duration ratios were calculated. Candidate motifs with scores higher than the default threshold values were retrieved as modified motifs. Those with infrequent occurrences $(<50$ and $<10$ in P-MAGs and V-MAGs, respectively) or very low methylation fractions $(<10 \%)$ in each MAG were excluded from further analysis. Motifs with several ambiguous sequences that were considered to have occurred by misdetection were manually curated. For example, HBNNNNNNVGGW $\underline{\mathbf{C}} \mathrm{CNH}$ was detected in CM1_5m.V59, where $\mathrm{H}=\mathrm{A} / \mathrm{C} / \mathrm{T}, \mathrm{B}=\mathrm{A} / \mathrm{G} / \mathrm{T}, \mathrm{V}=\mathrm{A} / \mathrm{C} / \mathrm{G}$, and $\mathrm{W}=\mathrm{A} / \mathrm{W}$, but this motif represents palindromic GGW $\underline{C}$ C and the spurious partial sequences of former HBNNNNNNV and latter NH were likely due to incomplete detection of the motif. Notably, we frequently found candidate motifs that showed such ambiguity in V-MAGs. This is likely a result of the weak motif estimation power from small genomes; the low presence of motifs in the genome negatively affected the motif-finding algorithm implemented in the MotifMaker tool, which is based on progressive testing for seeking longer motif sequences using a branch-and-bound search. 
Genes encoding DNA methyltransferases (MTases), restriction endonucleases (REases), and DNA sequence-recognition proteins (S subunits) were searched using BLASTP (35) against an experimentally confirmed gold-standard dataset from REBASE (43) (downloaded on February 9, 2021), with a cutoff e-value of $\leq 1 \mathrm{E}-5$. Sequence specificity information for each hit MTase gene was retrieved from REBASE. The flanking regions of the MTase genes were investigated to search for REase genes and to examine whether they constitute RM systems. The BREX (45) and DISARM (46) systems were sought based on Pfam domains.

For accurate analysis of methylome diversity, P-MAGs with $>20 \%$ completeness were used for the phylogenetic analysis. A maximum-likelihood (ML) tree of the MAGs was constructed using PhyloPhlan3 (99) on the basis of a set of 400 conserved prokaryotic marker genes (100) with "--force_nucleotides -diversity high --accurate' settings. The proteomic tree of V-MAGs was estimated using ViPTreeGen (101) with default settings.

For estimation of a robust phylogenetic tree of Alphaproteobacteria P-MAGs, those with higher quality (>25\% completeness) were retrieved and used for ML tree reconstruction using PhyloPhlan3 with '-force_nucleotides --diversity low --accurate' settings. To calculate the expected/observed (E/O) ratio of each motif sequence, the expected and observed counts of its presentation on the genome were computed using R'MES (102) and SeqKit (103), respectively. An ML tree of MTases was constructed using MEGA X (104) with LG substitution model with a gamma distribution $(\mathrm{LG}+\mathrm{G})$, which was selected based on the Bayesian information criterion (BIC), and 100 bootstrap replicates. Three pairs of the Proteobacteria genome and carried MTase homolog gene were retrieved from the NCBI database and REBASE, respectively, and used for outgroups; pairs of Campylobacter sp. RM16704 and M.Csp16704III, Haemophilus influenzae Rd KW20 and M.HinfI, and Helicobacter pylori 26695 and M.HpyAIV. Multiple sequence alignment was calculated using deposited genomes were referred to by Muñoz-Gómez et al. (54) and Haro-Moreno et al. (55) were retrieved from the NCBI database, respectively (Data S5). For the analysis of Alphaproteobacteria, four Betaproteobacteria and four Gammaproteobacteria genomes were retrieved from the NCBI database and used as outgroups. For the analysis of SAR11, genomes of Rickettsia felis URRWXCa12, Rhodospirillum rubrum ATCC11170, Rickettsia bellii RML369-C, and Acidiphilium cryptum JF-5 were retrieved from the NCBI database and used as outgroups. The phylogenetic trees were estimated using PhyloPhlan3 with '-force_nucleotides --diversity low --accurate' settings. Subclades of the SAR11 P-MAGs were inferred based on the topology of the phylogenetic tree according to the previous definition $(55,106,107)$. 


\section{Experimental verification of MTase activities}

To verify MTase specificity, selected MTase genes were artificially synthesized with codon optimization by Thermo Fisher Scientific (Data S4). The genes were cloned into the pCold III expression vector (Takara Bio, Shiga, Japan) using the In-Fusion HD Cloning Kit (Takara Bio). Additional specific sequences were inserted downstream of the termination codon for the methylation assay if an appropriate sequence was absent from the plasmid vector. The constructs were transformed into E. coli HST04 dam/dcm (Takara Bio), which lacks the dam and dcm MTase genes. In addition, constructs of Ct9H90mP5_10800 and Ct9H90mP30_5500 were alternatively induced into the pET-47b(+) expression vector (Merck KGaA) using the In-Fusion HD Cloning Kit and transformed into E. coli BL21 Star (DE3) (Thermo Fisher Scientific) due to severe insolubilization of the expressed protein in the former manner. Soluble protein levels were measured using SDS-PAGE analysis as needed. The E. coli strains were cultured in LB broth supplemented with the appropriate antibiotics, ampicillin or kanamycin. MTase expression was induced according to the supplier's protocol for the expression vector. Plasmid DNA was isolated using the FastGene Plasmid Mini Kit (Nippon Genetics, Tokyo, Japan) or NucleoSpin Plasmid EasyPure Kit (Takara Bio). The REase NdeI was employed for the linearization of plasmid DNAs. Methylation status was assayed simultaneously with linearizing digestion using the appropriate REases. All REases were purchased from New England BioLabs (NEB) (Ipswich, Massachusetts, USA). All digestion reactions were performed at $37^{\circ} \mathrm{C}$ for $1 \mathrm{~h}$, except for the simultaneous digestion of HinfI and TfiI at $37{ }^{\circ} \mathrm{C}$ for $30 \mathrm{~min}$, followed by $65^{\circ} \mathrm{C}$ for $30 \mathrm{~min}$.

We further verified MTases with novel motif specificities (i.e., Ct9H300mP26_1870, Ct9H90mP5_10800, CM1200mP2_32760,CM15mP129_7780, CM1200mP10_13750, and CM15mP20_30) by SMRT sequencing. Chromosomal DNA of E. coli HST04 dam/2 $\mathrm{cm}^{-}$strains in which target MTases were transformed were extracted using the DNeasy UltraClean Microbial Kit (QIAGEN) according to the supplier's protocol after induction of gene expression. Multiplex SMRT sequencing was conducted using PacBio Sequel II (Pacific Biosciences of California) according to the manufacturer's standard protocols. Briefly, 12-50 kb DNA fragments from each genomic DNA sample were extracted using the BluePippin size selection system (Sage Science) for continuous long read (CLR) sequencing. SMRT sequencing libraries were prepared using the SMRTbell Express Template Prep Kit 2.0 and Barcoded Overhang Adapter Kit 8A, according to the manufacturer's protocol (Pacific Biosciences of California). All final SMRT libraries were sequenced using a Sequel II SMRT Cell 8M. Methylated motifs were detected using SMRT Link v9.0 against the E. coli K-12 MG1655 reference genome (RefSeq NC_000913.2). 
For the in vitro assay of CM15mP111_3240 MTase and its point mutant, recombinant proteins were purified. N-terminal 6xHis-tag fusion MTase and D49G mutant were constructed using PCR and cloned into the pCold III expression vector. E. coli cells (HST04 $\mathrm{dam}^{-} / \mathrm{dcm}^{-}$) transformed with the constructs were grown at $37{ }^{\circ} \mathrm{C}$ for $16 \mathrm{~h}$ in $20 \mathrm{~mL}$ of medium A (LB medium containing $50 \mu \mathrm{g} / \mathrm{mL}$ of ampicillin) with shaking. The culture was then inoculated into $2 \mathrm{~L}$ of medium A in a 5-L flask, incubated at $37{ }^{\circ} \mathrm{C}$ for $2-3 \mathrm{~h}$ with shaking, and grown until the optical density at $\mathrm{A}_{600} \mathrm{~nm}$ reached 1.0. Then, MTase expression was induced at $15{ }^{\circ} \mathrm{C}$ with the addition of $0.1 \mathrm{mM}$ isopropyl $\beta$-d-1-thiogalactopyranoside (IPTG), and the cultures were subsequently incubated for $16 \mathrm{~h}$ according to the manufacturer's standard protocol of pCold. E. coli cells were lysed by sonication in Buffer A [20 mM HEPES-Na (pH 7.5), $150 \mathrm{mM} \mathrm{NaCl}, 5 \%$ glycerol, $1 \mathrm{mM}$ DTT, and $50 \mathrm{mM}$ imidazole]. The cell lysate was centrifuged at $12,000 \mathrm{rpm}$ for $30 \mathrm{~min}$ at $4{ }^{\circ} \mathrm{C}$ and passed through a GD/X syringe filter with a $0.45-\mu \mathrm{m}$ pore size (Cytiva, Marlborough, Massachusetts, USA). The supernatant was subjected to a two-column chromatography using ÄKTA prime chromatography system (Cytiva). The presence of the desired protein was confirmed using SDS-PAGE. The sample was loaded onto a 5-mL HisTrap HP column (Cytiva) at a flow rate of $2 \mathrm{~mL} / \mathrm{min}$. The column was then washed with Buffer A. The His-tagged protein was eluted with Buffer B [20 mM HEPES-Na (pH 7.5), $150 \mathrm{mM} \mathrm{NaCl}, 5 \%$ glycerol, $1 \mathrm{mM}$ DTT, and $300 \mathrm{mM}$ imidazole]. The eluted fractions were pooled and diluted 5-fold with Buffer C [20 mM HEPES-Na ( $\mathrm{pH} 7.5), 150 \mathrm{mM} \mathrm{NaCl}$, and $1 \mathrm{mM}$ DTT]. The diluted solution was concentrated to approximately $5 \mathrm{~mL}$ using $30 \mathrm{kDa}$ molecular weight cutoff Amicon Ultra centrifugal filters (Merck KGaA), passed through a Millex-GP syringe filter with $0.22 \mu \mathrm{m}$ pore size (Merck KGaA), and loaded onto a HiLoad 16/600 Superdex 200 pg column (Cytiva) pre-equilibrated with Buffer C. The protein was collected as a single peak and concentrated to $2.5 \mathrm{mg} / \mathrm{mL}$ ( $\sim 50 \mu \mathrm{M}$ in monomer concentration). The protein was aliquoted, flash-frozen in liquid nitrogen, and stored at $-80{ }^{\circ} \mathrm{C}$, or preserved with $50 \%$ glycerol at $-30{ }^{\circ} \mathrm{C}$ until used for further assays. $\mathrm{Na}$ pH 7.5, $100 \mathrm{mM} \mathrm{NaCl}$, and $100 \mu \mathrm{g} / \mathrm{mL}$ BSA] with $5 \mathrm{nM}$ substrate DNA and $1 \mu \mathrm{M}$ purified MTase in solution at $20^{\circ} \mathrm{C}$ for $1 \mathrm{~h}$ unless specified otherwise. To investigate salt sensitivity, the $\mathrm{NaCl}$ concentration was

Purified MTases were used for enzymatic methylation. The substrate unmethylated DNAs were produced using PCR with the pCold III vector transferred CM15mP111_3240 gene as a template to match with the in vivo assay of MTase. Methylation reactions were carried out in a reaction buffer [20 mM HEPESvaried from 0-400 $\mathrm{mM}$. To investigate the thermal sensitivity, the reaction temperature was varied from 5$40{ }^{\circ} \mathrm{C}$. For investigations of star activity, MTase and glycerol concentrations were varied between 1 and 15 $\mu \mathrm{M}$ and $0-10 \% \mathrm{v} / \mathrm{v}$, respectively, and the reaction time was extended to $3 \mathrm{~h}$. The reactions were started with $160 \mu \mathrm{M}$ SAM (NEB) in solution and stopped by adding guanidinium thiocyanate solution buffer NTI (Takara 
794 Bio). After the methylation reaction following DNA purification, the methylation status was assayed using

795 HinfI digestion at $37^{\circ} \mathrm{C}$ for $30 \mathrm{~min}$.

Data availability

The raw sequencing data and assembled genomes were deposited in the DDBJ Sequence Read Archive and DDBJ/ENA/GenBank, respectively (Data S6). All data are registered under BioProject ID PRJDB11069 [http://trace.ddbj.nig.ac.jp/BPSearch/bioproject?acc=PRJDB11069].

\section{References}

803 1. A. W. Struck, M. L. Thompson, L. S. Wong, J. Micklefield, S-adenosyl-methionine-dependent methyltransferases: Highly versatile enzymes in biocatalysis, biosynthesis and other biotechnological applications. ChemBioChem. 13, 2642-2655 (2012). P. Weigele, E. A. Raleigh, Biosynthesis and function of modified bases in bacteria and their viruses. Chem. Rev. 116, 12655-12687 (2016).

4. J. Casadesús, in Advances in Experimental Medicine and Biology, A. Jeltsch, R. Z. Jurkowska, Eds. (Springer International Publishing, Cham, 2016; https://doi.org/10.1007/978-3-319-43624-1_3), vol. 945, pp. 35-61.

S. S. Mohapatra, E. G. Biondi, in Cellular Ecophysiology of Microbe, T. Krell, Ed. (Springer International Publishing, Cham, 2017; https://doi.org/10.1007/978-3-319-20796-4_23-1), pp. 1-21.

6. X. Zhou, J. Wang, J. Herrmann, W. E. Moerner, L. Shapiro, Asymmetric division yields progeny cells with distinct modes of regulating cell cycle-dependent chromosome methylation. Proc. Natl. Acad. Sci. 116, 15661-15670 (2019).

7. J. B. Kozdon, M. D. Melfi, K. Luong, T. A. Clark, M. Boitano, S. Wang, B. Zhou, D. Gonzalez, J. Collier, S. W. Turner, J. Korlach, L. Shapiro, H. H. McAdams, Global methylation state at base-pair resolution of the Caulobacter genome throughout the cell cycle. Proc. Natl. Acad. Sci. U. S. A. 110, E4658-E4667 (2013).

K. Gärtner, S. Klähn, S. Watanabe, S. Mikkat, I. Scholz, W. R. Hess, M. Hagemann, Cytosine N4methylation via M.Ssp6803II is involved in the regulation of transcription, fine-tuning of DNA 
replication and DNA repair in the cyanobacterium Synechocystis sp. PCC 6803. Front. Microbiol. 10, 1233 (2019).

9. I. Vandenbussche, A. Sass, M. Pinto-Carbó, O. Mannweiler, L. Eberl, T. Coenye, DNA methylation epigenetically regulates gene expression in Burkholderia cenocepacia and controls biofilm formation, cell aggregation, and motility. mSphere. 5, e00455-20 (2020).

10. P. H. Oliveira, J. W. Ribis, E. M. Garrett, D. Trzilova, A. Kim, O. Sekulovic, E. A. Mead, T. Pak, S. Zhu, G. Deikus, M. Touchon, M. Lewis-Sandari, C. Beckford, N. E. Zeitouni, D. R. Altman, E. Webster, I. Oussenko, S. Bunyavanich, A. K. Aggarwal, A. Bashir, G. Patel, F. Wallach, C. Hamula, S. Huprikar, E. E. Schadt, R. Sebra, H. van Bakel, A. Kasarskis, R. Tamayo, A. Shen, G. Fang, Epigenomic characterization of Clostridioides difficile finds a conserved DNA methyltransferase that mediates sporulation and pathogenesis. Nat. Microbiol. 5, 166-180 (2020).

11. T. M. Nye, K. M. Jacob, E. K. Holleyid, J. M. Nevarez, S. Dawidid, L. A. Simmons, M. E. Watson, DNA methylation from a Type I restriction modification system influences gene expression and virulence in Streptococcus pyogenes. PLoS Pathog. 15, e1007841 (2019).

12. O. Mannweiler, M. Pinto-Carbó, M. Lardi, K. Agnoli, L. Eberl, An investigation of Burkholderia cepacia complex methylomes via SMRT sequencing and mutant analysis. J. Bacteriol. (2021), doi:10.1128/JB.00683-20.

13. A. S. Ershova, I. S. Rusinov, S. A. Spirin, A. S. Karyagina, A. V. Alexeevski, Role of restrictionmodification systems in prokaryotic evolution and ecology. Biochem. 80, 1373-1386 (2015).

14. A. J. Harris, A. D. Goldman, The complex phylogenetic relationships of a 4mC/6mA DNA methyltransferase in prokaryotes. Mol. Phylogenet. Evol. 149, 106837 (2020).

15. L. Wang, S. Jiang, Z. Deng, P. C. Dedon, S. Chen, DNA phosphorothioate modification-a new multifunctional epigenetic system in bacteria. FEMS Microbiol. Rev. 43, 109-122 (2019).

16. Y. N. Srikhanta, K. L. Fox, M. P. Jennings, The phasevarion: Phase variation of type III DNA methyltransferases controls coordinated switching in multiple genes. Nat. Rev. Microbiol. 8, 196 (2010).

17. Y. Furuta, I. Kobayashi, Mobility of DNA sequence recognition domains in DNA methyltransferases suggests epigenetics-driven adaptive evolution. Mob. Genet. Elements. 2, 292-296 (2012).

18. M. A. Sánchez-Romero, J. Casadesús, The bacterial epigenome. Nat. Rev. Microbiol. 18, 7-20 (2020).

19. I. A. Murray, T. A. Clark, R. D. Morgan, M. Boitano, B. P. Anton, K. Luong, A. Fomenkov, S. W. Turner, J. Korlach, R. J. Roberts, The methylomes of six bacteria. Nucleic Acids Res. 40, 11450-11462 (2012).

20. M. J. Blow, T. A. Clark, C. G. Daum, A. M. Deutschbauer, A. Fomenkov, R. Fries, J. Froula, D. D. Kang, R. R. Malmstrom, R. D. Morgan, J. Posfai, K. Singh, A. Visel, K. Wetmore, Z. Zhao, E. M. 
Rubin, J. Korlach, L. A. Pennacchio, R. J. Roberts, The epigenomic landscape of prokaryotes. PLoS Genet. 12, e1005854 (2016).

21. B. M. Forde, L. J. McAllister, J. C. Paton, A. W. Paton, S. A. Beatson, SMRT sequencing reveals differential patterns of methylation in two O111:H- STEC isolates from a hemolytic uremic syndrome outbreak in Australia. Sci. Rep. 9, 9436 (2019).

22. N. A. Ahlgren, Y. Chen, D. M. Needham, A. E. Parada, R. Sachdeva, V. Trinh, T. Chen, J. A. Fuhrman, Genome and epigenome of a novel marine Thaumarchaeota strain suggest viral infection, phosphorothioation DNA modification and multiple restriction systems. Environ. Microbiol. 19, 24342452 (2017).

23. B. Cao, C. Chen, M. S. DeMott, Q. Cheng, T. A. Clark, X. Xiong, X. Zheng, V. Butty, S. S. Levine, G. Yuan, M. Boitano, K. Luong, Y. Song, X. Zhou, Z. Deng, S. W. Turner, J. Korlach, D. You, L. Wang, S. Chen, P. C. Dedon, Genomic mapping of phosphorothioates reveals partial modification of short consensus sequences. Nat. Commun. 5, 3951 (2014).

24. L. Xiong, S. Liu, S. Chen, Y. Xiao, B. Zhu, Y. Gao, Y. Zhang, B. Chen, J. Luo, Z. Deng, X. Chen, L. Wang, S. Chen, A new type of DNA phosphorothioation-based antiviral system in archaea. Nat. Commun. 10, 1688 (2019).

25. S. Jeudy, S. Rigou, J.-M. Alempic, J.-M. Claverie, C. Abergel, M. Legendre, The DNA methylation landscape of giant viruses. Nat. Commun. 11, 2657 (2019).

26. S. R. Coy, E. R. Gann, S. E. Papoulis, M. E. Holder, N. J. Ajami, J. F. Petrosino, E. R. Zinser, J. L. Van Etten, S. W. Wilhelm, SMRT sequencing of Paramecium bursaria Chlorella virus-1 reveals diverse methylation stability in adenines targeted by restriction modification systems. Front. Microbiol. 11, 887 (2020).

27. A. Rhoads, K. F. Au, PacBio sequencing and its applications. Genomics. Proteomics Bioinformatics. 13, 278-289 (2015).

28. E. B. Fichot, R. S. Norman, Microbial phylogenetic profiling with the Pacific Biosciences sequencing platform. Microbiome. 1, 10 (2013).

29. S. Hiraoka, Y. Okazaki, M. Anda, A. Toyoda, S. Nakano, W. Iwasaki, Metaepigenomic analysis reveals the unexplored diversity of DNA methylation in an environmental prokaryotic community. Nat. Commun. 10, 159 (2019).

30. E. L. Moss, D. G. Maghini, A. S. Bhatt, Complete, closed bacterial genomes from microbiomes using nanopore sequencing. Nat. Biotechnol. 38, 701-707 (2020).

31. K. Hanawa, H. Mitsudera, Variation of water system distribution in the Sanriku Coastal Area. J. Oceanogr. 42, 435-446 (1986). 
32. P. Menzel, K. L. Ng, A. Krogh, Fast and sensitive taxonomic classification for metagenomics with Kaiju. Nat. Commun. 7, 11257 (2016).

33. R. Agarwala, T. Barrett, J. Beck, D. A. Benson, C. Bollin, E. Bolton, D. Bourexis, J. R. Brister, S. H. Bryant, K. Canese, C. Charowhas, K. Clark, M. DiCuccio, I. Dondoshansky, M. Feolo, K. Funk, L. Y. Geer, V. Gorelenkov, W. Hlavina, M. Hoeppner, B. Holmes, M. Johnson, V. Khotomlianski, A. Kimchi, M. Kimelman, P. Kitts, W. Klimke, S. Krasnov, A. Kuznetsov, M. J. Landrum, D. Landsman, J. M. Lee, D. J. Lipman, Z. Lu, T. L. Madden, T. Madej, A. Marchler-Bauer, I. Karsch-Mizrachi, T. Murphy, R. Orris, J. Ostell, C. O’Sullivan, V. Palanigobu, A. R. Panchenko, L. Phan, K. D. Pruitt, K. Rodarmer, W. Rubinstein, E. W. Sayers, V. Schneider, C. L. Schoch, G. D. Schuler, S. T. Sherry, K. Sirotkin, K. Siyan, D. Slotta, A. Soboleva, V. Soussov, G. Starchenko, T. A. Tatusova, K. Todorov, B. W. Trawick, D. Vakatov, Y. Wang, M. Ward, W. J. Wilbur, E. Yaschenko, K. Zbicz, Database resources of the National Center for Biotechnology Information. Nucleic Acids Res. 45, D12-D17 (2017).

34. M. G. Pachiadaki, J. M. Brown, J. Brown, O. Bezuidt, P. M. Berube, S. J. Biller, N. J. Poulton, M. D. Burkart, J. J. La Clair, S. W. Chisholm, R. Stepanauskas, Charting the complexity of the marine microbiome through single-cell genomics. Cell. 179, 1623-1635.e11 (2019).

35. C. Camacho, G. Coulouris, V. Avagyan, N. Ma, J. Papadopoulos, K. Bealer, T. L. Madden, BLAST+: architecture and applications. BMC Bioinformatics. 10, 421 (2009).

P. Yilmaz, L. W. Parfrey, P. Yarza, J. Gerken, E. Pruesse, C. Quast, T. Schweer, J. Peplies, W. Ludwig, F. O. Glöckner, The SILVA and "All-species Living Tree Project (LTP)" taxonomic frameworks. Nucleic Acids Res. 42, D643-D648 (2014).

38. K. D. Weynberg, M. J. Allen, W. H. Wilson, Marine prasinoviruses and their tiny plankton hosts: A review. Viruses. 9, 43 (2017).

S. J. Low, M. Džunková, P.-A. Chaumeil, D. H. Parks, P. Hugenholtz, Evaluation of a concatenated protein phylogeny for classification of tailed double-stranded DNA viruses belonging to the order Caudovirales. Nat. Microbiol. 4, 1306-1315 (2019).

\section{S. Sunagawa, L. P. Coelho, S. Chaffron, J. R. Kultima, K. Labadie, G. Salazar, B. Djahanschiri, G.} Zeller, D. R. Mende, A. Alberti, F. M. Cornejo-Castillo, P. I. Costea, C. Cruaud, F. D’Ovidio, S. Engelen, I. Ferrera, J. M. Gasol, L. Guidi, F. Hildebrand, F. Kokoszka, C. Lepoivre, G. Lima-Mendez, J. Poulain, B. T. Poulos, M. Royo-Llonch, H. Sarmento, S. Vieira-Silva, C. Dimier, M. Picheral, S. Searson, S. Kandels-Lewis, C. Bowler, C. de Vargas, G. Gorsky, N. Grimsley, P. Hingamp, D. Iudicone, O. Jaillon, F. Not, H. Ogata, S. Pesant, S. Speich, L. Stemmann, M. B. Sullivan, J. Weissenbach, P. Wincker, E. Karsenti, J. Raes, S. G. Acinas, P. Bork, E. Boss, C. Bowler, M. Follows, L. Karp-Boss, U. Krzic, E. G. Reynaud, C. Sardet, M. Sieracki, D. Velayoudon, Structure and function of the global ocean microbiome. Science (80-. ). 348, 1261359 (2015). 
bioRxiv preprint doi: https:/doi.org/10.1101/2021.05 08.442635. this version posted May 8,2021 . The copyright holder for this preprint (which was not certified by peer review) is the author/funder, who has granted bioRxiv a license to display the preprint in perpetuity. It is made available under aCC-BY-NC-ND 4.0 International license.

40. F. Flaviani, D. Schroeder, C. Balestreri, J. Schroeder, K. Moore, K. Paszkiewicz, M. Pfaff, E. Rybicki, A pelagic microbiome (iruses to protists) from a small cup of seawater. Viruses. 9, 47 (2017).

41. A. C. Gregory, A. A. Zayed, N. Conceição-Neto, B. Temperton, B. Bolduc, A. Alberti, M. Ardyna, K. Arkhipova, M. Carmichael, C. Cruaud, C. Dimier, G. Domínguez-Huerta, J. Ferland, S. Kandels, Y. Liu, C. Marec, S. Pesant, M. Picheral, S. Pisarev, J. Poulain, J.-É. Tremblay, D. Vik, M. Babin, C. Bowler, A. I. Culley, C. de Vargas, B. E. Dutilh, D. Iudicone, L. Karp-Boss, S. Roux, S. Sunagawa, P. Wincker, M. B. Sullivan, S. G. Acinas, M. Babin, P. Bork, E. Boss, C. Bowler, G. Cochrane, C. de Vargas, M. Follows, G. Gorsky, N. Grimsley, L. Guidi, P. Hingamp, D. Iudicone, O. Jaillon, S. Kandels-Lewis, L. Karp-Boss, E. Karsenti, F. Not, H. Ogata, S. Pesant, N. Poulton, J. Raes, C. Sardet, S. Speich, L. Stemmann, M. B. Sullivan, S. Sunagawa, P. Wincker, Marine DNA viral macro- and microdiversity from pole to pole. Cell. 177, 1109-1123.e14 (2019).

42. T. Alarcón-Schumacher, S. Guajardo-Leiva, J. Antón, B. Díez, Elucidating viral communities during a phytoplankton bloom on the west Antarctic Peninsula. Front. Microbiol. 10, 1014 (2019).

43. R. J. Roberts, T. Vincze, J. Posfai, D. Macelis, REBASE - a database for DNA restriction and modification: Enzymes, genes and genomes. Nucleic Acids Res. 43, D298-D299 (2015).

44. S. J. Giovannoni, SAR11 bacteria: The most abundant plankton in the oceans. Ann. Rev. Mar. Sci. 9, 231-255 (2017).

45. T. Goldfarb, H. Sberro, E. Weinstock, O. Cohen, S. Doron, Y. Charpak-Amikam, S. Afik, G. Ofir, R. Sorek, BREX is a novel phage resistance system widespread in microbial genomes. EMBO J. 34, 169183 (2015).

46. G. Ofir, S. Melamed, H. Sberro, Z. Mukamel, S. Silverman, G. Yaakov, S. Doron, R. Sorek, DISARM is a widespread bacterial defence system with broad anti-phage activities. Nat. Microbiol. 3, 90-98 (2018).

47. A. Mouammine, J. Collier, The impact of DNA methylation in Alphaproteobacteria. Mol. Microbiol. 110, 1-10 (2018).

48. D. Gonzalez, J. B. Kozdon, H. H. McAdams, L. Shapiro, J. Collier, The functions of DNA methylation by CcrM in Caulobacter crescentus: A global approach. Nucleic Acids Res. 42, 3720-3735 (2014).

49. A. G. Davis-Richardson, J. T. Russell, R. Dias, A. J. McKinlay, R. Canepa, J. R. Fagen, K. T. Rusoff, J. C. Drew, B. Kolaczkowski, D. W. Emerich, E. W. Triplett, Integrating DNA methylation and gene expression data in the development of the soybean-Bradyrhizobium N2-fixing symbiosis. Front. Microbiol. 7, 518 (2016).

50. J. G. Borgaro, N. Benner, Z. Zhu, Fidelity undex determination of DNA methyltransferases. PLoS One. 8, e63866 (2013).

51. H. M. Cohen, D. S. Tawfik, A. D. Griffiths, Promiscuous methylation of non-canonical DNA sites by HaeIII methyltransferase. Nucleic Acids Res. 30, 3880-3885 (2002). 
bioRxiv preprint doi: https:/doi.org/10.1101/2021.05 08.442635. this version posted May 8,2021 . The copyright holder for this preprint (which was not certified by peer review) is the author/funder, who has granted bioRxiv a license to display the preprint in perpetuity. It is made available under aCC-BY-NC-ND 4.0 International license.

52. R. Wright, C. Stephens, L. Shapiro, The CcrM DNA methyltransferase is widespread in the alpha subdivision of proteobacteria, and its essential functions are conserved in Rhizobium meliloti and Caulobacter crescentus. J. Bacteriol. 179, 5869-5877 (1997).

53. J. R. Horton, C. B. Woodcock, S. B. Opot, N. O. Reich, X. Zhang, X. Cheng, The cell cycle-regulated DNA adenine methyltransferase CcrM opens a bubble at its DNA recognition site. Nat. Commun. 10, 4600 (2019).

54. S. A. Muñoz-Gómez, S. Hess, G. Burger, B. F. Lang, E. Susko, C. H. Slamovits, A. J. Roger, An updated phylogeny of the Alphaproteobacteria reveals that the parasitic Rickettsiales and Holosporales have independent origins. Elife. 8, e42535 (2019).

55. J. M. Haro-Moreno, F. Rodriguez-Valera, R. Rosselli, F. Martinez-Hernandez, J. J. Roda-Garcia, M. L. Gomez, O. Fornas, M. Martinez-Garcia, M. López-Pérez, Ecogenomics of the SAR11 clade. Environ. Microbiol. 22, 1748-1763 (2020).

56. N. G. Walworth, D. A. Hutchins, E. Dolzhenko, M. D. Lee, F. Fu, A. D. Smith, E. A. Webb, Biogeographic conservation of the cytosine epigenome in the globally important marine, nitrogenfixing cyanobacterium Trichodesmium. Environ. Microbiol. 19, 4700-4713 (2017).

57. I. M. Rambo, A. Marsh, J. F. Biddle, Cytosine methylation within marine sediment microbial communities: Potential epigenetic adaptation to the environment. Front. Microbiol. 10, 1291 (2019).

58. Y. Suzuki, S. Nishijima, Y. Furuta, J. Yoshimura, W. Suda, K. Oshima, M. Hattori, S. Morishita, Long-read metagenomic exploration of extrachromosomal mobile genetic elements in the human gut. Microbiome. 7, 119 (2019).

59. V. Somerville, S. Lutz, M. Schmid, D. Frei, A. Moser, S. Irmler, J. E. Frey, C. H. Ahrens, Long-read based de novo assembly of low-complexity metagenome samples results in finished genomes and reveals insights into strain diversity and an active phage system. BMC Microbiol. 19, 143 (2019).

60. Y. Furuta, H. Namba-Fukuyo, T. F. Shibata, T. Nishiyama, S. Shigenobu, Y. Suzuki, S. Sugano, M. Hasebe, I. Kobayashi, Methylome diversification through changes in DNA methyltransferase sequence specificity. PLoS Genet. 10, e1004272 (2014).

61. J. Beaulaurier, S. Zhu, G. Deikus, I. Mogno, X.-S. Zhang, A. Davis-Richardson, R. Canepa, E. W. Triplett, J. J. Faith, R. Sebra, E. E. Schadt, G. Fang, Metagenomic binning and association of plasmids with bacterial host genomes using DNA methylation. Nat. Biotechnol. 36, 61 (2017).

62. A. Tourancheau, E. A. Mead, X. S. Zhang, G. Fang, Discovering and exploiting multiple types of DNA methylation from individual bacteria and microbiome using nanopore sequencing. bioRxiv (2020), doi:10.1101/2020.02.18.954636.

63. E. G. Wilbanks, H. Doré, M. H. Ashby, C. Heiner, J. A. Eisen, Metagenomic methylation patterns resolve complex microbial genomes. bioRxiv (2021), doi:10.1101/2021.01.18.427177. 
bioRxiv preprint doi: https:/doi.org/10.1101/2021.05 08.442635. this version posted May 8,2021 . The copyright holder for this preprint (which was not certified by peer review) is the author/funder, who has granted bioRxiv a license to display the preprint in perpetuity. It is made available under aCC-BY-NC-ND 4.0 International license.

64. K. K. Kojima, Y. Furuta, K. Yahara, M. Fukuyo, Y. Shiwa, S. Nishiumi, M. Yoshida, T. Azuma, H. Yoshikawa, I. Kobayashi, Population evolution of Helicobacter pylori through diversification in DNA methylation and interstrain sequence homogenization. Mol. Biol. Evol. 33, 2848-2859 (2016).

65. M. S. Fullmer, M. Ouellette, A. S. Louyakis, R. T. Papke, J. P. Gogarten, The patchy distribution of restriction-modification system genes and the conservation of orphan methyltransferases in halobacteria. Genes (Basel). 10, 233 (2019).

66. M. M. Ashcroft, B. M. Forde, M. D. Phan, K. M. Peters, L. W. Roberts, K. G. Chan, T. M. Chong, W. F. Yin, D. L. Paterson, T. R. Walsh, M. A. Schembri, S. A. Beatson, Strain and lineage-level methylome heterogeneity in the multi-drug resistant pathogenic Escherichia coli ST101 clone. bioRxiv (2020), doi:10.1101/2020.06.07.138552.

67. I. Mruk, I. Kobayashi, To be or not to be: Regulation of restriction-modification systemas and other toxin-antitoxin systems. Nucleic Acids Res. 42, 70-86 (2014).

68. E. Teira, R. Logares, A. Gutiérrez-Barral, I. Ferrera, M. M. Varela, X. A. G. Morán, J. M. Gasol, Impact of grazing, resource availability and light on prokaryotic growth and diversity in the oligotrophic surface global ocean. Environ. Microbiol. 21, 1482-1496 (2019).

69. D. P. Häder, R. P. Sinha, Solar ultraviolet radiation-induced DNA damage in aquatic organisms: Potential environmental impact. Mutat. Res. 571, 221-233 (2005).

70. M. Middelboe, C. P. D. Brussaard, Marine viruses: Key players in marine ecosystems. Viruses. 9, 302 (2017).

71. W. H. Wilson, J. L. Van Etten, M. J. Allen, in Current Topics in Microbiology and Immunology, J. L. Van Etten, Ed. (Springer Berlin Heidelberg, Berlin, Heidelberg, 2009; https://doi.org/10.1007/978-3540-68618-7_1), vol. 328, pp. 1-42.

72. J. Murphy, J. Mahony, S. Ainsworth, A. Nauta, D. van Sinderen, Bacteriophage orphan DNA methyltransferases: Insights from their bacterial origin, function, and occurrence. Appl. Environ. Microbiol. 79, 7547-7555 (2013).

73. A. Kęsik-Szeloch, Z. Drulis-Kawa, B. Weber-Dąbrowska, J. Kassner, G. Majkowska-Skrobek, D. Augustyniak, M. Łusiak-Szelachowska, M. Zaczek, A. Górski, A. M. Kropinski, Characterising the biology of novel lytic bacteriophages infecting multidrug resistant Klebsiella pneumoniae. Virol. J. 10, 100 (2013).

74. N. Sternberg, J. Coulby, Cleavage of the bacteriophage P1 packaging site (pac) is regulated by adenine methylation. Proc. Natl. Acad. Sci. 87, 8070-8074 (1990).

75. D. G. Scraba, R. D. Bradley, M. Leyritz-Wills, R. A. J. Warren, Bacteriophage $\varphi$ W-14: The contribution of covalently bound putrescine to DNA packing in the phage head. Virology. 124, 152 160 (1983). 
bioRxiv preprint doi: https://doi.org/10.1101/2021.05.08.442635. this version posted May 8,2021 . The copyright holder for this preprint (which was not certified by peer review) is the author/funder, who has granted bioRxiv a license to display the preprint in perpetuity. It is made available under aCC-BY-NC-ND 4.0 International license.

76. M. B. Lobocka, D. J. Rose, G. Plunkett, M. Rusin, A. Samojedny, H. Lehnherr, M. B. Yarmolinsky, F. R. Blattner, Genome of bacteriophage P1. J. Bacteriol. 186, 7032-7068 (2004).

77. M. Brilli, M. Fondi, R. Fani, A. Mengoni, L. Ferri, M. Bazzicalupo, E. G. Biondi, The diversity and evolution of cell cycle regulation in alpha-proteobacteria: a comparative genomic analysis. BMC Syst. Biol. 4, 52 (2010).

78. C. P. D. Brussaard, Optimization of procedures for counting viruses by flow Cytometry. Appl. Environ. Microbiol. 70, 1506-1513 (2004).

79. P. A. del Giorgio, D. F. Bird, Y. T. Prairie, D. Planas, Flow cytometric determination of bacterial abundance in lake plankton with the green nucleic acid stain SYTO 13. Limnol. Oceanogr. 41, 783789 (1996).

80. M. Hirai, S. Nishi, M. Tsuda, M. Sunamura, Y. Takaki, T. Nunoura, Library construction from subnanogram DNA for pelagic sea water and deep-sea sediments. Microbes Environ. 32, 336-343 (2017).

81. L. M. Rodriguez-R, S. Gunturu, J. M. Tiedje, J. R. Cole, K. T. Konstantinidis, Nonpareil 3: Fast estimation of metagenomic coverage and sequence diversity. mSystems. 3, e00039-18 (2018).

82. E. Kopylova, L. Noé, H. Touzet, SortMeRNA: Fast and accurate filtering of ribosomal RNAs in metatranscriptomic data. Bioinformatics. 28, 3211-3217 (2012).

83. K. Lagesen, P. Hallin, E. A. Rødland, H.-H. Stærfeldt, T. Rognes, D. W. Ussery, RNAmmer: Consistent and rapid annotation of ribosomal RNA genes. Nucleic Acids Res. 35, 3100-3108 (2007).

84. C. Quast, E. Pruesse, P. Yilmaz, J. Gerken, T. Schweer, P. Yarza, J. Peplies, F. O. Glöckner, The SILVA ribosomal RNA gene database project: Improved data processing and web-based tools. Nucleic Acids Res. 41, D590-D596 (2013).

85. D. Hyatt, G.-L. Chen, P. LoCascio, M. Land, F. Larimer, L. Hauser, Prodigal: Prokaryotic gene recognition and translation initiation site identification. BMC Bioinformatics. 11, 119 (2010).

86. T. Magoč, S. L. Salzberg, FLASH: fast length adjustment of short reads to improve genome assemblies. Bioinformatics. 27, 2957-2963 (2011).

87. J. Ruan, H. Li, Fast and accurate long-read assembly with wtdbg2. Nat. Methods. 17, 155-158 (2020).

88. R. Kundu, J. Casey, W.-K. Sung, HyPo: Super fast \& accurate polisher for long read genome assemblies. bioRxiv (2019), doi:10.1101/2019.12.19.882506.

89. H. Li, Minimap2: Pairwise alignment for nucleotide sequences. Bioinformatics. 34, 3094-3100 (2018).

90. B. Langmead, S. L. Salzberg, Fast gapped-read alignment with Bowtie 2. Nat. Methods. 9, 357-359 (2012).

91. D. D. Kang, J. Froula, R. Egan, Z. Wang, MetaBAT, an efficient tool for accurately reconstructing single genomes from complex microbial communities. PeerJ. 3, e1165 (2015). 
92. D. H. Parks, M. Imelfort, C. T. Skennerton, P. Hugenholtz, G. W. Tyson, CheckM: Assessing the quality of microbial genomes recovered from isolates, single cells, and metagenomes. Genome Res. 25, 1043-1055 (2015).

93. R. M. Bowers, N. C. Kyrpides, R. Stepanauskas, M. Harmon-Smith, D. Doud, T. B. K. Reddy, F. Schulz, J. Jarett, A. R. Rivers, E. A. Eloe-Fadrosh, S. G. Tringe, N. N. Ivanova, A. Copeland, A. Clum, E. D. Becraft, R. R. Malmstrom, B. Birren, M. Podar, P. Bork, G. M. Weinstock, G. M. Garrity, J. A. Dodsworth, S. Yooseph, G. Sutton, F. O. Glöckner, J. A. Gilbert, W. C. Nelson, S. J. Hallam, S. P. Jungbluth, T. J. G. Ettema, S. Tighe, K. T. Konstantinidis, W.-T. Liu, B. J. Baker, T. Rattei, J. A. Eisen, B. Hedlund, K. D. McMahon, N. Fierer, R. Knight, R. Finn, G. Cochrane, I. Karsch-Mizrachi, G. W. Tyson, C. Rinke, N. C. Kyrpides, L. Schriml, G. M. Garrity, P. Hugenholtz, G. Sutton, P. Yilmaz, F. Meyer, F. O. Glöckner, J. A. Gilbert, R. Knight, R. Finn, G. Cochrane, I. Karsch-Mizrachi, A. Lapidus, F. Meyer, P. Yilmaz, D. H. Parks, A. Murat Eren, L. Schriml, J. F. Banfield, P. Hugenholtz, T. Woyke, T. G. S. Consortium, Minimum information about a single amplified genome (MISAG) and a metagenome-assembled genome (MIMAG) of bacteria and archaea. Nat. Biotechnol. 35, 725-731 (2017).

94. I. T. Fiddes, J. Armstrong, M. Diekhans, S. Nachtweide, Z. N. Kronenberg, J. G. Underwood, D. Gordon, D. Earl, T. Keane, E. E. Eichler, D. Haussler, M. Stanke, B. Paten, Comparative Annotation Toolkit (CAT)-simultaneous clade and personal genome annotation. Genome Res. 28, 1029-1038 (2018).

95. J. Mistry, R. D. Finn, S. R. Eddy, A. Bateman, M. Punta, Challenges in homology search: HMMER3 and convergent evolution of coiled-coil regions. Nucleic Acids Res. 41, e121 (2013).

96. R. D. Finn, P. Coggill, R. Y. Eberhardt, S. R. Eddy, J. Mistry, A. L. Mitchell, S. C. Potter, M. Punta, M. Qureshi, A. Sangrador-Vegas, G. A. Salazar, J. Tate, A. Bateman, The Pfam protein families database: Towards a more sustainable future. Nucleic Acids Res. 44, D279-D285 (2016).

97. J. Guo, B. Bolduc, A. A. Zayed, A. Varsani, G. Dominguez-Huerta, T. O. Delmont, A. A. Pratama, M. C. Gazitúa, D. Vik, M. B. Sullivan, S. Roux, VirSorter2: a multi-classifier, expert-guided approach to detect diverse DNA and RNA viruses. Microbiome. 9, 37 (2021).

98. S. Nayfach, A. P. Camargo, F. Schulz, E. Eloe-Fadrosh, S. Roux, N. C. Kyrpides, CheckV assesses the quality and completeness of metagenome-assembled viral genomes. Nat. Biotechnol. (2020), doi:10.1038/s41587-020-00774-7.

99. F. Asnicar, A. M. Thomas, F. Beghini, C. Mengoni, S. Manara, P. Manghi, Q. Zhu, M. Bolzan, F. Cumbo, U. May, J. G. Sanders, M. Zolfo, E. Kopylova, E. Pasolli, R. Knight, S. Mirarab, C. Huttenhower, N. Segata, Precise phylogenetic analysis of microbial isolates and genomes from metagenomes using PhyloPhlAn 3.0. Nat. Commun. 11, 2500 (2020). 
100. N. Segata, D. Börnigen, X. C. Morgan, C. Huttenhower, PhyloPhlAn is a new method for improved phylogenetic and taxonomic placement of microbes. Nat. Commun. 4, 2304 (2013).

101. Y. Nishimura, T. Yoshida, M. Kuronishi, H. Uehara, H. Ogata, S. Goto, ViPTree: The viral proteomic tree server. Bioinformatics. 33, 2379-2380 (2017).

102. S. Schbath, M. Hoebeke, in Advances in Genomic Sequence Analysis and Pattern Discovery (WORLD SCIENTIFIC, 2011; https://doi.org/10.1142/9789814327732_0002), vol. Volume 7 of Science, Engineering, and Biology Informatics, pp. 25-64.

103. W. Shen, S. Le, Y. Li, F. Hu, SeqKit: A Cross-Platform and Ultrafast Toolkit for FASTA/Q File Manipulation. PLoS One. 11, e0163962 (2016).

104. S. Kumar, G. Stecher, M. Li, C. Knyaz, K. Tamura, MEGA X: Molecular evolutionary genetics analysis across computing platforms. Mol. Biol. Evol. 35, 1547-1549 (2018).

105. F. Sievers, A. Wilm, D. Dineen, T. J. Gibson, K. Karplus, W. Li, R. Lopez, H. McWilliam, M. Remmert, J. Söding, J. D. Thompson, D. G. Higgins, Fast, scalable generation of high-quality protein multiple sequence alignments using Clustal Omega. Mol. Syst. Biol. 7, 539 (2011).

106. S. Kraemer, A. Ramachandran, D. Colatriano, C. Lovejoy, D. A. Walsh, Diversity and biogeography of SAR11 bacteria from the Arctic Ocean. ISME J. 14, 79-90 (2020).

107. D. Tsementzi, J. Wu, S. Deutsch, S. Nath, L. M. Rodriguez-R, A. S. Burns, P. Ranjan, N. Sarode, R. R. Malmstrom, C. C. Padilla, B. K. Stone, L. A. Bristow, M. Larsen, J. B. Glass, B. Thamdrup, T. Woyke, K. T. Konstantinidis, F. J. Stewart, SAR11 bacteria linked to ocean anoxia and nitrogen loss. Nature. 536, 179-183 (2016).

\section{Acknowledgments}

We would like to thank the captain, crew, and onboard scientists and technicians of the R/V Kaimei (JAMSTEC) during KM19-07 cruise. The SMRT and Nanopore sequencing were supported by NIG. We thank Keiko Tanaka, Eiji Tasumi, Akiko Makabe, Minoru Hamana, Masahito Shigemitsu, Hiroshi Uchida, Yusuke Tsukatani, Hidetaka Nomaki, Takeuchi Akinori, Shuhei Ota, Yuya Tada, Mancha Mabaso, Jarishma Gokul, and Thulani Makhalanyane for seawater sampling. We are grateful to Masami Koizumi for technical assistance with cell and viral-like particle counting and flow cytometry experiments, and Fumie Kondo and Miwako Tsuda for their helpful suggestions and support in the molecular experiments. This work was financially supported by the Japan Society for the Promotion of Science (grant numbers JP18K11636, JP19H04246, JP19H05667, JP19H05684, JP19K21203, JP20H02020, and JP20K15444), and the Institute for Fermentation, Osaka (IFO). 


\section{Author contributions}

1131 SH conceived and designed the study, performed the sampling, molecular experiments,

1132 bioinformatics analyses, and wrote the manuscript. TS performed the sampling, designed and performed the

1133 molecular experiments and protein purification, and wrote the manuscript. MH performed the sampling and

1134 DNA sequencing using Illumina. AT performed DNA sequencing using PacBio and Nanopore. SK designed

1135 the cruise. TY designed and performed sampling and wrote the manuscript. TN wrote the manuscript and

1136 supervised the project. All authors read and approved the final manuscript.

\section{Competing interests}

1139 The authors declare no competing interests.

$1141 \quad$ Figures 

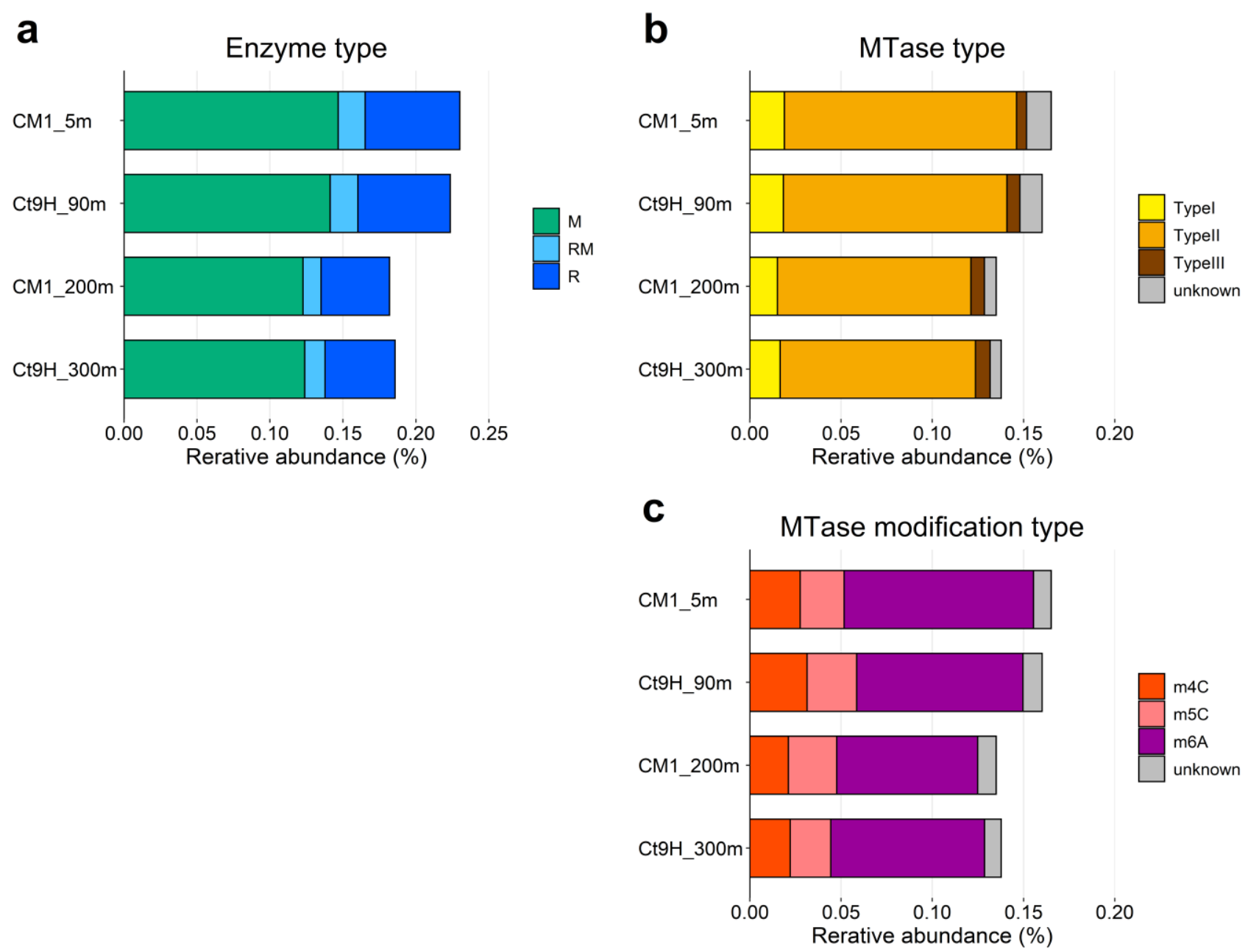

1143 Fig. 1. Relative abundances of DNA restriction and modification enzyme genes. CDSs predicted from HiFi 1144 reads were used in this analysis. a Distribution of RM types: DNA methyltransferase (MTase; M), Restriction 1145 endonuclease (REase; R), and protein fused with M and R domains (RM). b Distribution of MTase types. c 1146 Distribution of modification types. 
bioRxiv preprint doi: https://doi.org/10.1101/2021.05.08 442635. this version posted May 8, 2021. The copyright holder for this preprint (which was not certified by peer review) is the author/funder, who has granted bioRxiv a license to display the preprint in perpetuity. It is made available under aCC-BY-NC-ND 4.0 International license.
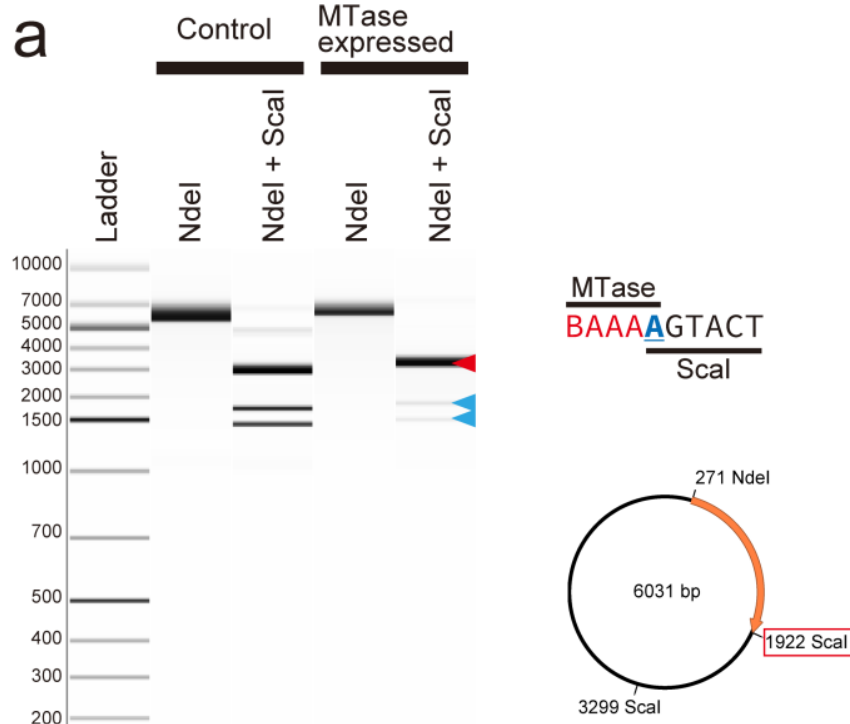

C
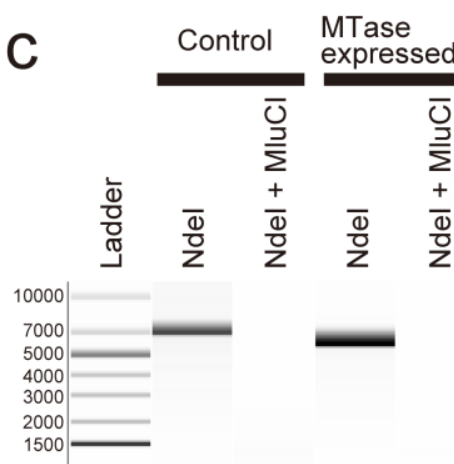

\begin{tabular}{l}
\hline 1000 \\
700 \\
500 \\
\hline 400 \\
300 \\
200 \\
\hline 75
\end{tabular}
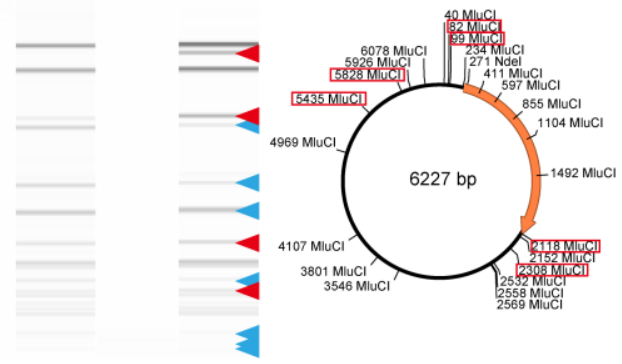

e
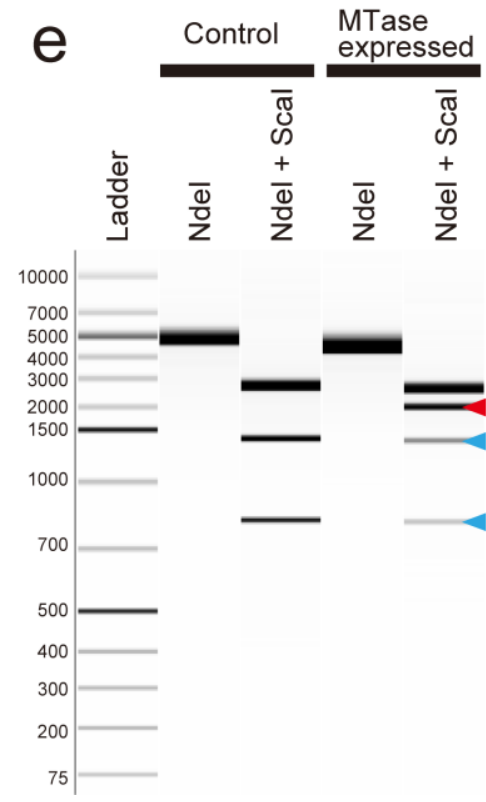

MTase

$5^{\prime}$-GGAGTA $\overline{\overline{C T C C}}-3^{\prime}$ $3^{\prime}$-CCTCATGAGG-5'

MTase Scal

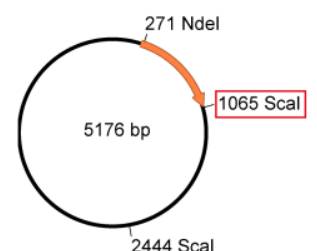

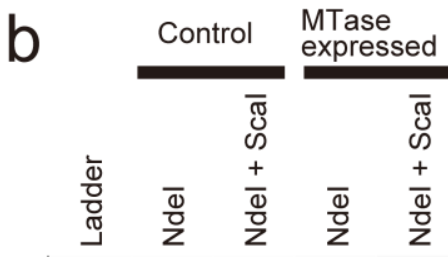
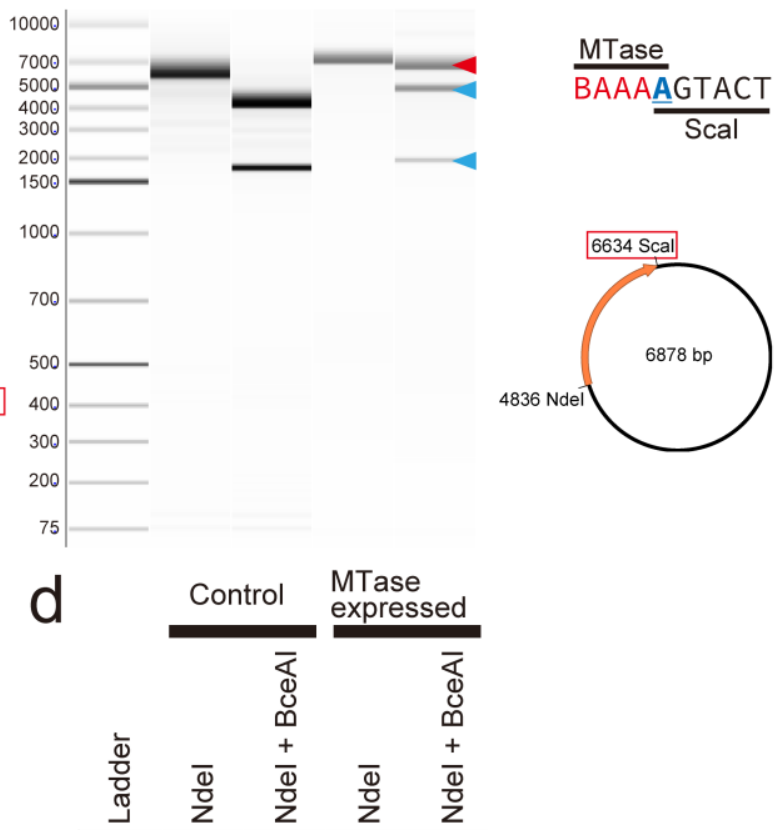

MTase

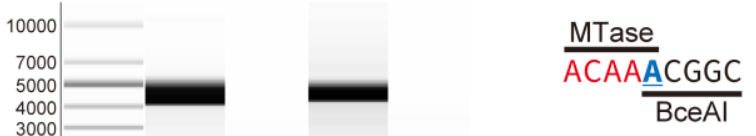

3000
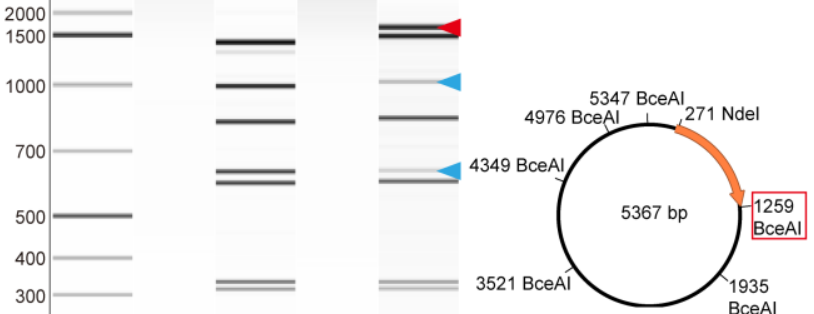
1149 Fig. 2. REase digestion assays of MTases with novel specificity. a Assay of the Ct9H300mP26_1870 gene.

1150 ScaI was used, where the plasmid contained two AGTACT target sites. Within the two sites, one of the target 1151 sites was BAAAAGTACT, where overlapped BAAAA and AGTACT were recognized by the MTase and 1152 REase. The schematic representation and plasmid map are presented on the right side. The orange arrow 1153 represents the transferred gene, and the digestion sites with a red frame represent the location of the 1154 overlapped sequence. b Assay of the Ct9H90mP5_10800 gene. ScaI was used, where the plasmid contained 1155 one AGTACT target site in the BAAAAGTACT site. c Assay of the CM1200mP2_32760 gene. MluCI were 1156 used, where the plasmid contained 23 AATT target sites. Within them, the six target sites were CAAATT, 1157 where overlapped CAAAT and AATT were recognized by the MTase and REase, respectively. d Assay of the 1158 CM15mP129_7780 gene. BceI were used, where the plasmid contained six ACGGC target sites. Within them, 1159 one of the target sites was ACAAACGGC, where overlapped ACAAA and ACGGC were recognized by the 1160 MTase and REase, respectively. e Assay of the CM1200mP10_13750 gene. ScaI were used, where the 1161 plasmid contained two TCATGA target sites. Within them, one of the target sites was GGAGTACTCC, 1162 where a pair of CTCC and GGAG (comprehensive sequence of CTCC) and TCATGA were recognized by the 1163 MTase and REase, respectively. The pCold III (a,c-e) and pET-47b(+) (e) were used as expression vectors. 1164 The band sizes were logically expected to emerge (red triangles) and reduce (blue triangles) when the induced 1165 MTase causes methylation. All plasmid DNAs were linearized using NdeI. 


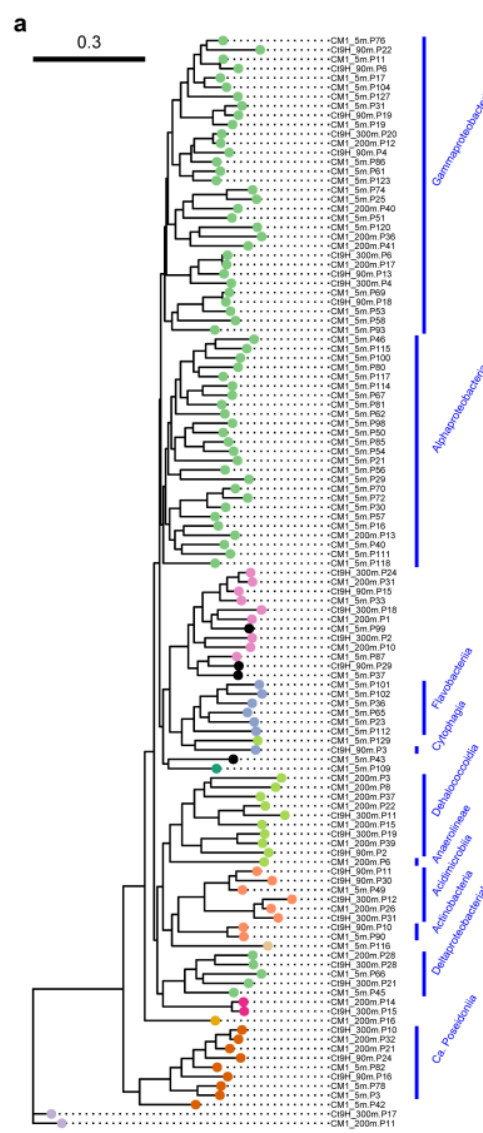

1167

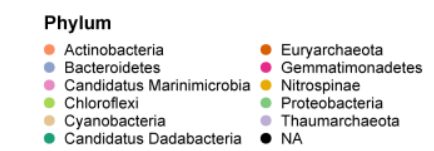

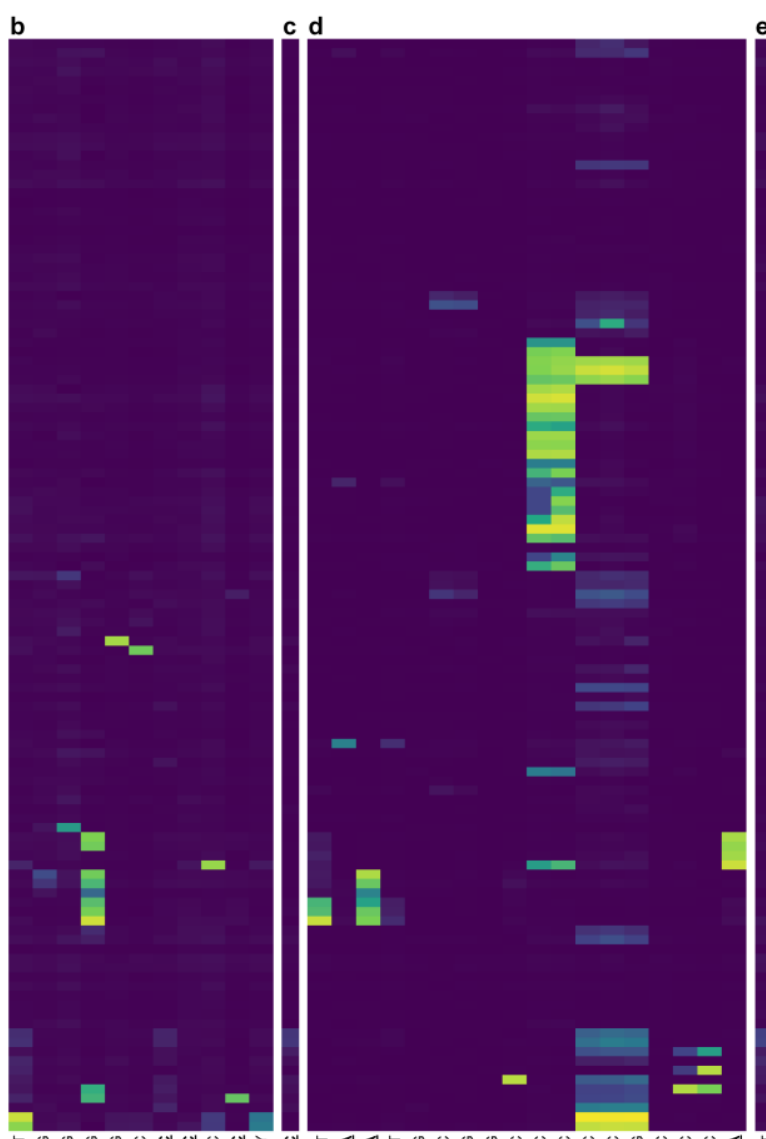
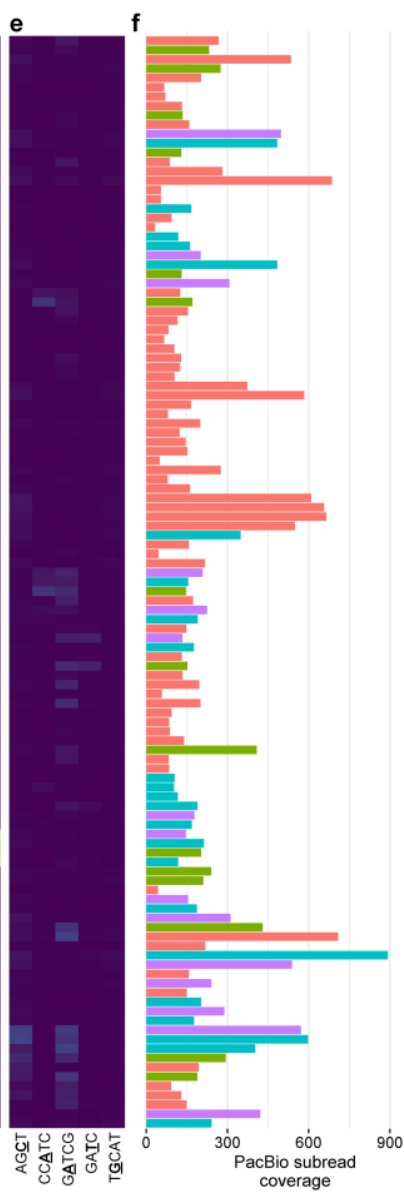

$\mathrm{m} 4 \mathrm{C}$

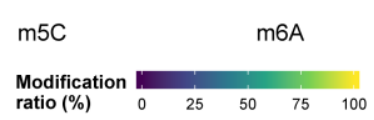

Site Unclassified $\mathrm{CM} 1-5 \mathrm{~m}$
$\mathrm{C} 9 \mathrm{H}-90 \mathrm{~m}$
$\mathrm{CM} 1-200 \mathrm{~m}$
$\mathrm{C}+9 \mathrm{H}_{-} 300 \mathrm{~m}$

1168 Fig. 3. Methylomes of P-MAGs. a A phylogenetic tree was constructed using a set of up to 400 conserved

1169 bacterial marker genes via the maximum-likelihood method. Node color indicates taxonomy at the phylum

1170 level. Nodes were grouped at class to family levels if estimated (blue bars and texts). b,c,d,e Modification

1171 ratios of detected motifs per genome. $\mathbf{b} \mathrm{m} 4 \mathrm{C}, \mathbf{c} \mathrm{m} 5 \mathrm{C}, \mathbf{d} \mathrm{m} 6 \mathrm{~A}$, and e unclassified modifications were

1172 individually shown. Motifs detected from P-MAGs without spurious sequence were used. The color range

1173 from blue over green to yellow represents modification ratios of motifs on each genome. We should note that

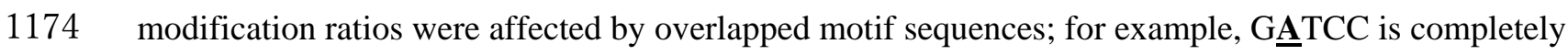

1175 overlapped by GATC, and both motifs showed similar modification rates in their genomes except in

1176 Gammaproteobacteria CM1_5m.P58 where GㅁTCC was detected on the genome from the metaepigenomic

1177 analysis and concordantly the modification ratio of GATCC was higher than that of GATC. $\mathbf{f}$ Coverages of

1178 subread on each genome. The bar color represents the source sample of the genome. 
a

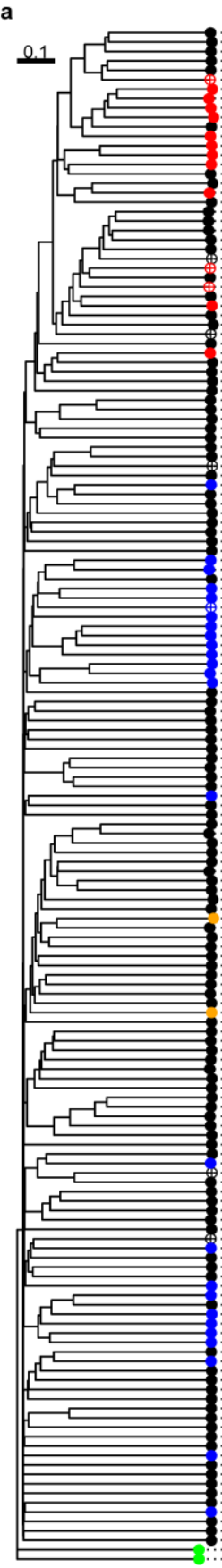

Classification

- NA Podoviridae

- Myoviridae Phycodnaviridae

b

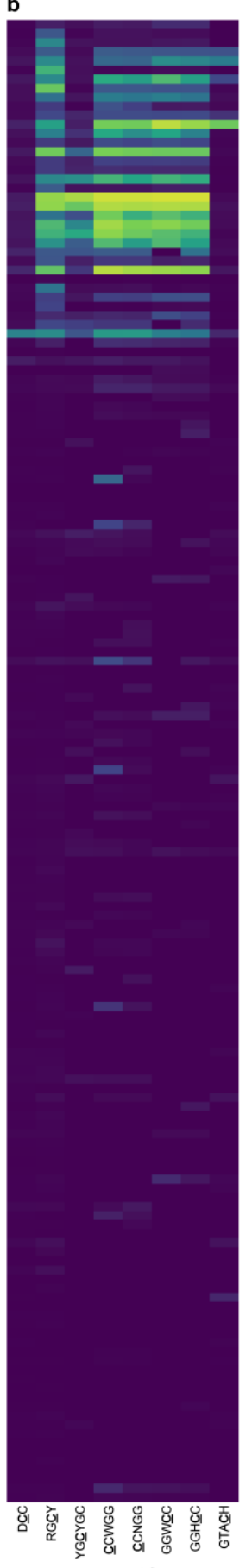

c
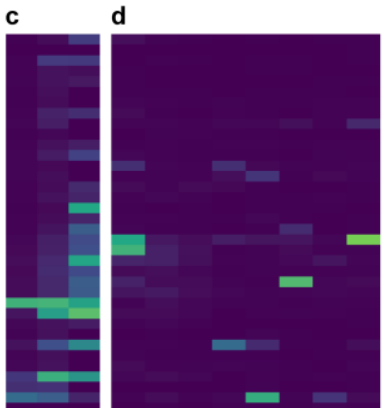

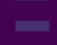
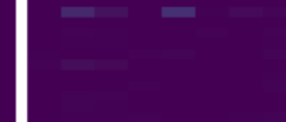

1181 Fig. 4. Methylomes of V-MAGs. a A proteomic tree was generated based on the global genomic similarities 1182 between viral genomes. Proviruses are indicated by circle cross. Node color indicates taxonomy at the family 1183 level. b,c,d Modification ratios of $\mathbf{b}$ m4C, $\mathbf{c}$ m5C, and $\mathbf{d}$ m6A motifs are presented individually. e Coverages 1184 of subread on each genome. See Fig. 3. 
a

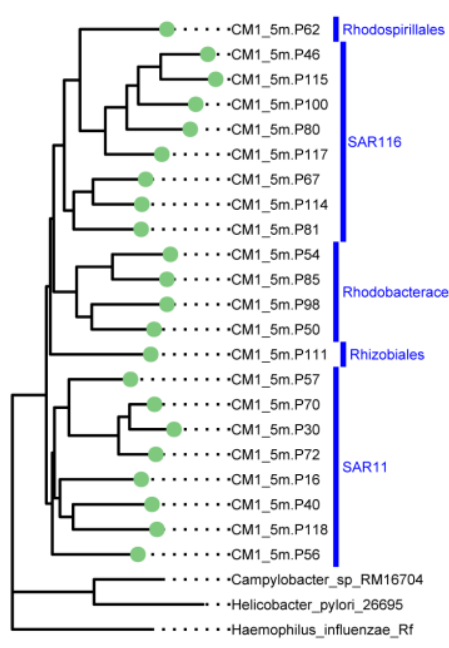

1186 b

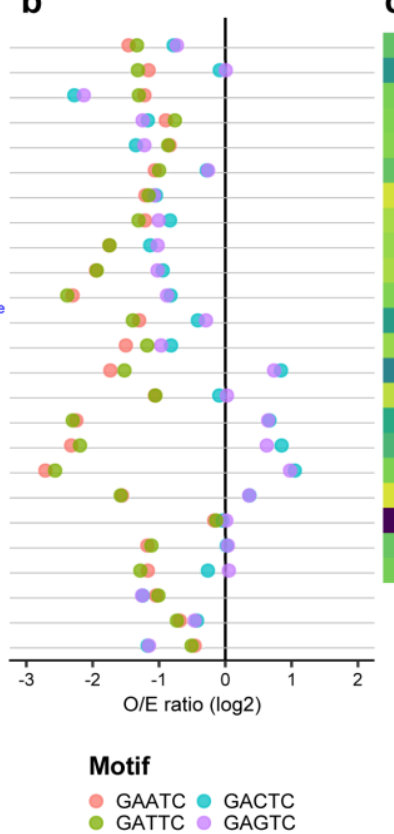

C

Modification ratio (\%)

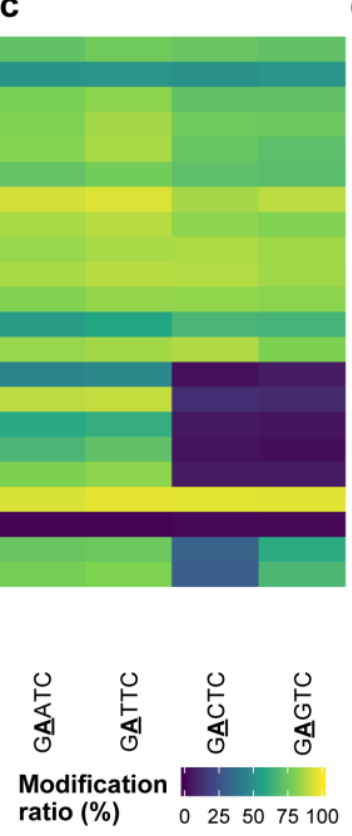

d

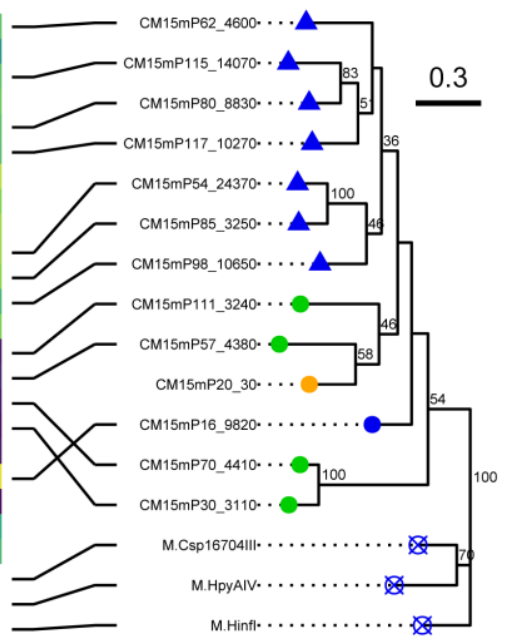

Sequence State

GANTC Possibly active GADTC
GAWTC Experimentive

Fig. 5. Methylomes and phylogenetic analysis of Alphaproteobacteria P-MAGs. Three homolog MTases, which were found in Proteobacteria isolates and previously confirmed to recognize GANTC were retrieved from REBASE and used as outgroups in this analysis; M.HpyAIV from Helicobacter pylori (Epsilonproteobacteria), M.Csp16704III from Campylobacter sp. (Epsilonproteobacteria), and M.HinfI from Haemophilus influenzae (Gammaproteobacteria). P-MAGs with >25\% completeness were used in this analysis for robust phylogenetic tree prediction. a A phylogenetic tree of the Alphaproteobacteria P-MAGs. b Observed/Expected (O/E) ratio of the GANTC member. A pair of GAATC and GATTC sequences constitutes GAWTC, and all the four sequences (GAATC, GATTC, GACTC, and GAGTC) constituted the GANTC motif, where $\mathrm{W}=\mathrm{A} / \mathrm{T}$ and $\mathrm{N}=\mathrm{A} / \mathrm{C} / \mathrm{G} / \mathrm{T}$. $\mathbf{c}$ Modification ratios of each G $\underline{\mathrm{ANTC}}$ component. Blank rows indicate the outgroup whose methylation data was not available. $\mathbf{d}$ Phylogenetic tree of the MTase genes. Supporting bootstrap values greater than $40 \%$ are shown. The node shapes indicate MTases that were estimated (rectangle nodes) or experimentally confirmed (circle) whose specificity and outgroups were indicated by circle cross. The node colors indicate the specificity of each MTase. 